\title{
An In Vivo Biocatalytic Cascade Featuring an Artificial Enzyme Catalyzed New-to-Nature Reaction
}

\author{
Linda Ofori Atta ${ }^{[a] \dagger}$, Zhi Zhou [a]t‡ and Gerard Roelfes ${ }^{*[a]}$ \\ [a] L. Ofori Atta, Dr. Z. Zhou, Prof. Dr. G. Roelfes \\ Stratingh Institute for Chemistry, University of Groningen \\ Nijenborgh 4, 9747 AG Groningen (The Netherlands) \\ E-mail: j.g.roelfes@rug.nl \\ $\dagger$ These authors contributed equally. \\ $\ddagger$ current address: School of Pharmaceutical Sciences, Jiangnan University, Wuxi 214122, China.
}

\begin{abstract}
Artificial enzymes utilizing the genetically encoded nonproteinogenic amino acid p-aminophenylalanine (pAF) as catalytic residue are able to react with carbonyl compounds through an iminium ion mechanism, making reactions possible that have no equivalent in nature. Here, we report an in vivo biocatalytic cascade that is augmented with such an artificial enzyme catalyzed new-to-nature reaction. The artificial enzyme in this study is a pAF containing evolved variant of the Lactococcal multidrug resistance Regulator, designated LmrR_V15pAF_RMH, which efficiently converts in vivo produced benzaldehyde derivatives into the corresponding hydrazone products inside $E$. coli cells. These in vivo biocatalytic cascades comprising an artificial enzyme catalyzed reactions are an important step towards achieving a hybrid metabolism.
\end{abstract}

\section{Introduction}

The cellular metabolism uniquely demonstrates the power of biocatalytic cascade reactions in living cells. Molecular structures of remarkable complexity are produced using an intricate network system of interconnected biocatalytic reactions. Such biological systems have proven amenable to modification by metabolic engineering. ${ }^{[1]}$ However, the structural diversity that can be achieved is inherently limited by the synthetic repertoire available to nature. In contrast, chemical synthesis offers virtually unlimited versatility in reaction scope, but to date synthetic chemical systems cannot rival the sophistication of nature. Hence, an attractive solution would be to combine the best of both worlds to create a "hybrid metabolism", which augments biological synthesis with abiological catalytic chemistry. ${ }^{[2]}$

Most efforts to date have focused on supplementing biological synthesis with a transition metal- or organocatalysed catalysed reaction. ${ }^{[3-10]}$ While some encouraging results have been obtained, low activity of transition metal complexes and organocatalysts in biological systems, as well as potential mutual incompatibility, is often a limiting factor. An alternative approach involves using artificial enzymes, created by integrating a synthetic catalysts into a protein scaffold. ${ }^{[11]}$ The protein environment helps to accelerate the reaction by providing additional interactions, but also protects the chemical catalyst from the cellular environment. ${ }^{[2]}$ Recently, the first examples of application of artificial metalloenzymes in living cells were reported. ${ }^{[12-17]}$ Also, biocatalytic cascades involving an artificial metalloenzyme was reported, albeit that this was carried out in vitro, using isolated proteins. ${ }^{[18,19]}$ Finally, iridium-heme substituted P450 enzyme catalysed cyclopropanation of a biosynthetically produced terpene in a heavily engineered $E$. coli strain was recently reported. ${ }^{20]}$

We have recently introduced the concept of using genetically encoded non-canonical amino acids directly as organocatalytic residue in artificial enzymes. ${ }^{[21,22]}$ Here, we report the application of a catalytic p-aminophenylalanine (pAF) containing artificial enzyme in $E$. coli and its integration into an in vivo biocatalytic cascade. Thus biocatalytic cascades comprising natural enzymes, producing benzaldehyde derivatives from different starting materials, is augmented with an artificial enzyme catalyzed abiological reaction to create a hydrazone product.

The non-canonical amino acid p-aminophenyl alanine (pAF) into the Lactococcal multidrug resistance Regulator $(\mathrm{LmrR})^{[23]}$ using the amber stop codon suppression methodology. ${ }^{[21,24,25]}$ pAF contains an aniline side chain that can react with aldehydes to form transient iminium ion species that are versatile reactive intermediates in organocatalysis. ${ }^{[26]} \mathrm{LmrR}$ with $\mathrm{pAF}$ at position 15 , designated LmrR_V15pAF, was shown to catalyse the abiotic reaction of benzaldehyde derivatives with hydrazines to form hydrazones. ${ }^{[21,27,28]}$ Subsequent directed evolution gave rise to the variant with mutations A92R_N19M_F93H, designated LmrR_V15pAF_RMH, which showed a 57-fold increase in catalytic efficiency compared to the parent LmrR_V15pAF.[29] LmrR_V15pAF and evolved variants have also been used successfully in conjugate addition reactions to enals, also exploiting the iminium ion activation strategy. ${ }^{[30,31]}$ 


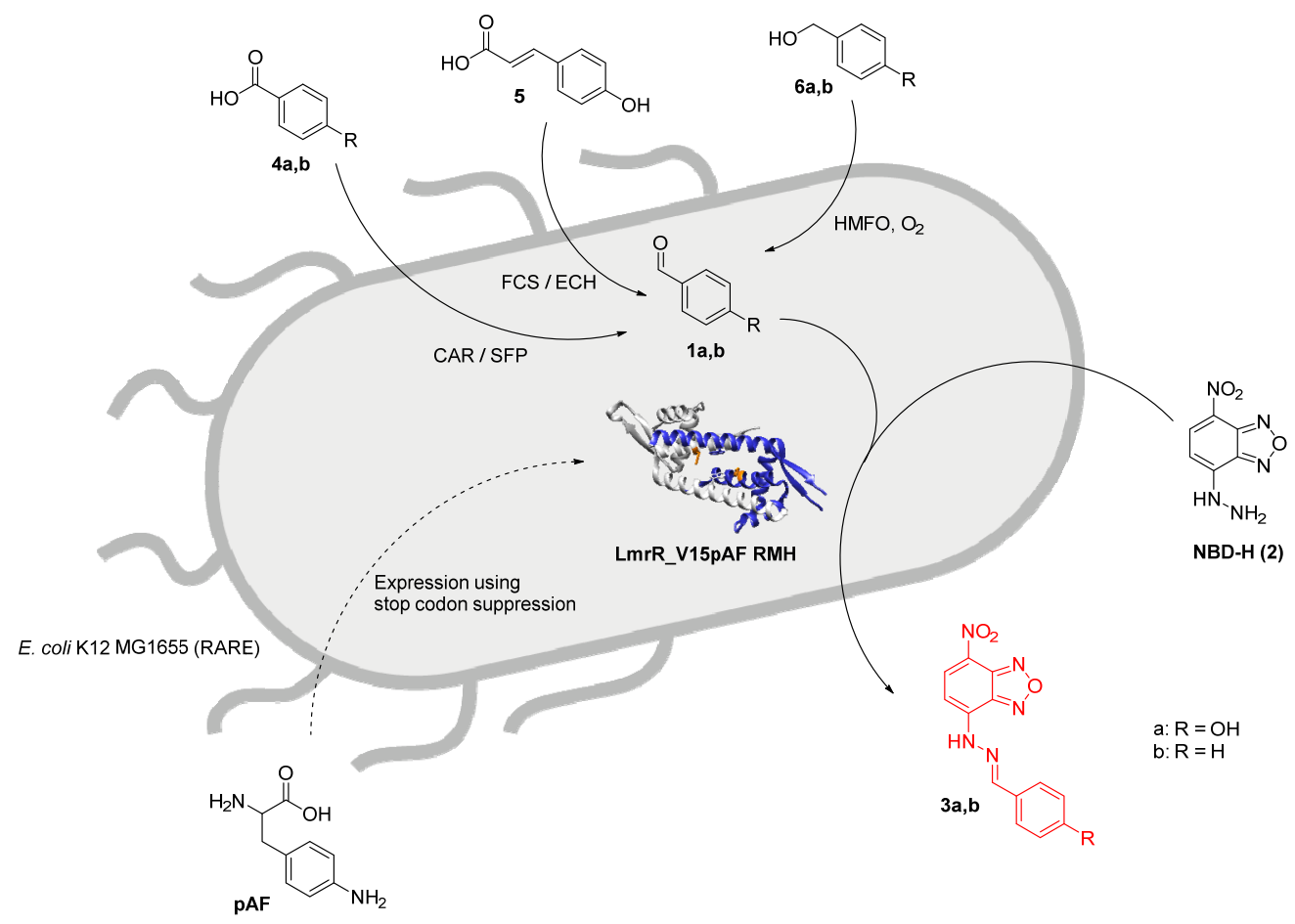

Scheme 1. Schematic representation of in vivo biocatalytic cascade featuring an abiological hydrazone formation reaction catalyzed by the designer enzyme LmrR_V15pAF_RMH.

Since LmrR_pAF is an artificial enzyme that is biosynthesized in living cells, we surmised that the catalysed hydrazone formation reaction could also be carried out in vivo. Moreover, since a variety of enzymatic synthesis of aldehydes from carboxylic acid or alcohol precursors are known, ${ }^{[32-34]}$ we envisioned an in vivo biocatalytic cascade comprising the biosynthesis of aldehydes using canonical enzymes followed by the LmrR_V15pAF_RMH catalyzed new-to-nature hydrazone formation reaction.

\section{Results and Discussion}

\section{Direct Incorporation of para-amino phenylalanine}

In our earlier work, it proved most efficient to introduce pAF indirectly, by first introducing $p$-azidophenylalanine (pAF) followed by Staudinger reduction with tris(2-carboxyethyl) phosphine (TCEP), added during the purifcation process. ${ }^{[21]}$ Direct incorporation of pAF by amber stop codon suppression, using the dedicated orthogonal translation system (pDULE2-paraaminoPhe), ${ }^{[25]}$ was possible, but proved to give significant misincorporation of other amino acids under standard expression conditions. However, for applications in vivo indirect incorporation via pAzF cannot be used. Hence, our initial effort focused on improving the direct incorporation of pAF by optimizing the expression conditions.

For this purpose, we tested the expression of the evolved variant of LmrR-V15pAF containing three mutations, N19M_A92R_F93H (LmrR_V15pAF_RMH). ${ }^{[29]}$ Additionally, the protein includes two mutations in the DNA binding domain, D55K_Q59K, and contains a C-terminal strep tag. Three different media, that are, Lysogeny Broth (LB), M9 minimal medium and minimal medium with vitamins (MMV), were evaluated for protein expression under different conditions.

In all cases, overexpression of full length protein was observed. MS analysis of the isolated proteins showed a mixture of the desired LmrR_V15pAF_RMH and variants resulting from mis-incorporation of, in particular, phenylalanine at position 15 , albeit with varying ratios depending on the conditions used. Judging from the relative intensities of the peaks, it was suggested that expression in MMV gave rise to the largest fraction of pAF incorporation. As a more quantitative measure for incorporation of $\mathrm{pAF}$, the kinetics of the catalyzed hydrazone formation reaction between p-hydroxybenzaldehyde (1a) and 4hydrazino-7-nitro-2,1,3-benzoxadiazole (NBD-H, 2) were determined and compared to those of LmrR_V15pAF_RMH prepared independently via indirect incorporation. ${ }^{[29]}$ The protein produced in LB and M9 media only showed low activity, suggesting that in these cases mostly misincorporation of other amino acids occurred, consistent with the MS results. In contrast the protein expressed in MMV medium at $24^{\circ} \mathrm{C}$ for 48 hours gave good activity. Based on the comparison of the catalytic efficiency it was concluded that the incorporation efficiency of pAF was $\sim 80 \%$ (Table S2). This was deemed sufficient for in vivo catalysis experiments. Additionally, the incorporation of pAF at position 15 was confirmed by trypsin digest (Figure S4). 


\section{Hydrazone formation reactions by an artificial enzyme in whole cells}

The activity of whole $E$. coli cells expressing wild type $L m r R$, i.e. without pAF, LmrR_V15pAF and LmrR_V15pAF_RMH from direct incorporation in the hydrazone formation reaction was tested by using exogeneous aldehydes and NBD-H. First, a set of $\mathrm{LmrR}$ variants were tested for the hydrazone formation from both para-hydroxybenzaldehyde (1a) and benzaldehyde (1b) in MMV and phosphate buffer $\mathrm{pH} 7$ for 3 hours in whole K-12 MG1655 (DE3) Cells. This strain allows for aromatic aldehyde accumulation because it has a reduced aromatic aldehyde reduction (RARE) activity. ${ }^{[35]}$ This is due to the fact that it has been engineered to reduce the reduction of aldehydes by endogenous ketoreductases and alcohol dehydrogenases. Control experiments without cells or with wild type LmrR gave up to $\sim 20 \%$ yield of the corresponding hydrazone products $\mathbf{3 a}$ and $\mathbf{3 b}$, respectively, in MMV medium, while only a low yield was observed in buffer (Figure 1). This shows that there is some background reaction in MMV medium. Using cells expressing

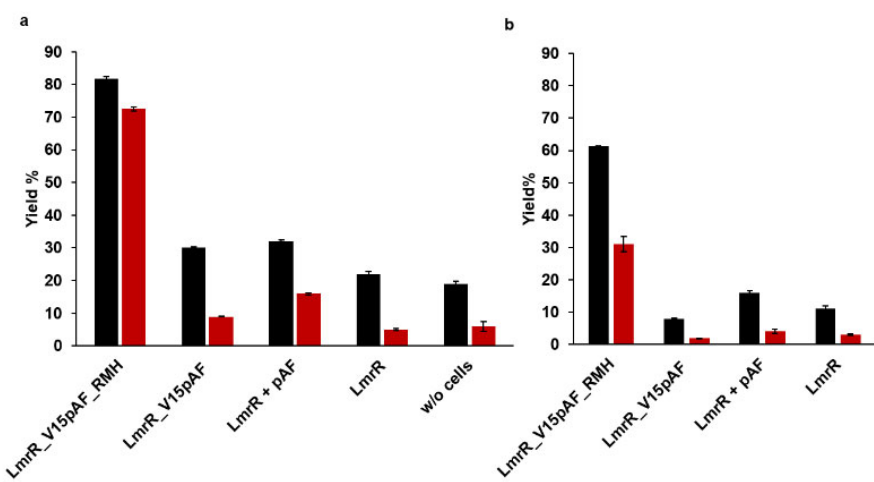

Figure 1. Yield of hydrazone products using MMV medium (black) and phosphate buffer (orange) in E.coli K12 MG1655 (RARE), (a) yield of 3b; conditions: $5 \mathrm{mM}$ 1b and $50 \mu \mathrm{M} 2$ in both MMV medium and phosphate buffer $\mathrm{pH} 7$, at $24^{\circ} \mathrm{C} / 135 \mathrm{rpm}$ for $3 \mathrm{~h}$. (b) yield of $3 \mathbf{a}$; conditions: $5 \mathrm{mM} 1 \mathrm{a}$ and $50 \mu \mathrm{M}$ 2 in both MMV medium and phosphate buffer $\mathrm{pH} 7$, at $24{ }^{\circ} \mathrm{C} / 135 \mathrm{rpm}$ for $3 \mathrm{~h}$. Error margins represent standard deviations from experiments performed in triplicate.

LmrR_V15pAF or LmrR supplemented with pAF showed a somewhat higher yield, indicating a catalytic effect, albeit quite small. In contrast, when using cells containing the evolved variant LmrR_V15pAF_RMH, the hydrazone products $\mathbf{3 a , b}$ were obtained with significantly higher yield of $62 \%$ and $82 \%$ yield in MMV and $31 \%$ and $73 \%$ in buffer, respectively. These results show that LmrR_V15pAF_RMH catalyzes the hydrazone formation in the cell. The lower yields obtained for $3 a$ reflect the lower reactivity of $\mathbf{1 a}$ due to the strongly electron donating $-\mathrm{OH}$ substituent.
Next, the biocatalytic synthesis of aromatic aldehydes in E.coli K12 MG1655 cells was investigated. We focused on production of benzaldehyde and para-hydroxy-benzaldehyde, using three different approaches:

i.

Carboxylic acid reductase (CAR) from Nocardia iwensis, along with its one time activator phosphopantetheinyl transferase (SFP) from Bacillus subtilis, reduces benzoic acids into their corresponding benzaldehydes in a NADPH and ATP dependent fashion. ${ }^{[36,37]}$ A codon optimised gene for CAR_SFP was harboured on a pETDuet1 vector.

ii. The conversion of p-coumaric acid to $p$ hydroxybenzaldehyde by a combination of feruloyl CoA synthase (encoded by Atfcs) and enoyl CoA hydratase/aldolase (encoded by Atech) from $A$. thermoflava $\mathrm{N} 1165{ }^{[38]}$ The conversion proceeds via the initial formation of the SCOA derivative by FCS, followed by the conjugate addition of water by $\mathrm{ECH} /$ hydratase and retro-aldol condensation by $\mathrm{ECH} /$ aldolase to produce the corresponding aldehyde. ${ }^{[39-41]}$ The required enzymes were expressed heterogeneously using a pETDuet-1 vector harbouring Atfcs_Atech.

iii. 5-hydroxymethylfurfural (HMF) oxidase (HMFO) from the white rot basidiomycete phanerochaete chrysosporium, an engineered flavin containing enzyme which uses molecular oxygen to oxidize alcohols to the corresponding carboxylic acids, via the intermediate formation of the corresponding aldehydes, ${ }^{[42,43]}$ with concomitant formation of $\mathrm{H}_{2} \mathrm{O}_{2}$. The HMFO gene was cloned into a pBAD vector.

The respective enzymes were expressed at $24^{\circ} \mathrm{C} / 135 \mathrm{rpm}$ for 16 hours in MMV medium, since this was the medium that gave the expression, the required substrates were added immediately to FCS-ECH (Scheme 1. 5) and CAR-SFP (Scheme 1. 4a,b) containing cells to a final concentration of $5 \mathrm{mM}$. For HMFO containing cells, $5 \mathrm{mM}$ substrate (Scheme 1. 6a,b), were added after 16 hours of expression and then reacted for $2 \mathrm{~h}$.

Both AtFcs-AtEch/E. coli RARE, starting from p-coumaric acid, and CAR-SFPIE. coli RARE, from p-hydroxybenzoic acid, produced a good yield of $\mathrm{p}$-hydroxybenzaldehyde. In contrast, the yield of the reduction of benzoic acid to benzaldehyde catalysed by CAR-SFPIE. coli RARE was significantly lower. FCS-ECH was not used for production of benzaldehyde, as these enzymes do not accept cinnamic acid as substrate. The whole cell HMFO 
catalysed oxidation of benzyl alcohol and p-hydroxybenzylalcohol gave $\mathbf{3 a}$ and $\mathbf{3 b}$ in 30 and $79 \%$ yield, respectively (Figure S11). Having demonstrated that both LmrR_V15pAF RMH catalyzed hydrazone formation and the production of its substrates benzaldehyde and p-hydroxybenzaldehyde can be achieved in $E$. coli RARE cells, we sought to combine these processes to create the in vivo biocatalytic cascade. Initial attempts to integrate the genes that encode CAR-SFP or FCS-ECH, LmrR_V15TAG and the orthogonal translation system on two plasmids proved unsuccessful due to the large size of CAR_SFP. For this reason, we then constructed the system by integrating three plasmids: pETDuet-1 harboring either the genes for CAR_SFP or FCS-ECH, pET28b + harbouring the gene for LmrR_V15pAF_RMH and pDULE-para-aminoPhe 2 containing the genes for the orthogonal translation system, p-aminoPhe tRNA synthetase/tRNA. The three plasmids were were co transformed into $E$. Coli $K-12$ MG1655 (RARE). HMFO, which is much smaller, and LmrR_V15pAF RMH were both cloned on pBAD. Hence, in this case a two plasmid system comprising the pBAD vector and pDULE-para- aminoPhe 2 could be used.

First, the different cell variants were tested in minimal media in the absence of NBD-H, to evaluate how the production of aldehyde is affected by the presence of all genes (Figure S3). Car-Sfp gave rise to a yield around $20 \%$ in both benzaldehyde and para hydroxy benzaldehyde production, which is lower than obtained before in the single plasmid system. In contrast, with the FCs-Ech containing cells the yield of $p$-hydroxy benzaldehyde was around $90 \%$. Finally, the oxidation of benzyl alcohols with the HMFO containing cells resulted in $60 \%$ yield of benzaldehyde and $\sim 50 \%$ of $p$-hydroxybenzaldehyde.

The in vivo cascade hydrazone formation were performed using cells from a $2.5 \mathrm{~mL}$ cell culture that were resuspended in freshly prepared MMV medium and the cascade reaction was started by adding NBD-H (final concentration $50 \mu \mathrm{M}$ ) and aldehyde precursor (final concentration $5 \mathrm{mM}$ ). Then the mixture was incubated at $24{ }^{\circ} \mathrm{C}$ in a shaker at $135 \mathrm{rpm}$ for 3 to 24 hours. The whole cells expressing both CAR with LmrR_V15pAF_RMH showed good reactivity with a hydrazone yield of $57 \%$ after 3 hours in whole cells. The control experiments with only LmrR_V15pAF_RMH showed no reactivity, which was expected because no aldehyde was produced. Surprisingly, using only CAR, in the absence of LmrR_V15pAF_RMH, resulted in $28 \%$ yield of the hydrazone. This suggests that CAR also to a small extent can catalyze hydrazone formation, albeit that this needs to be investigated further. p-hydroxybenzoic acid as substrate also gave rise to formation of the corresponding hydrazone product.
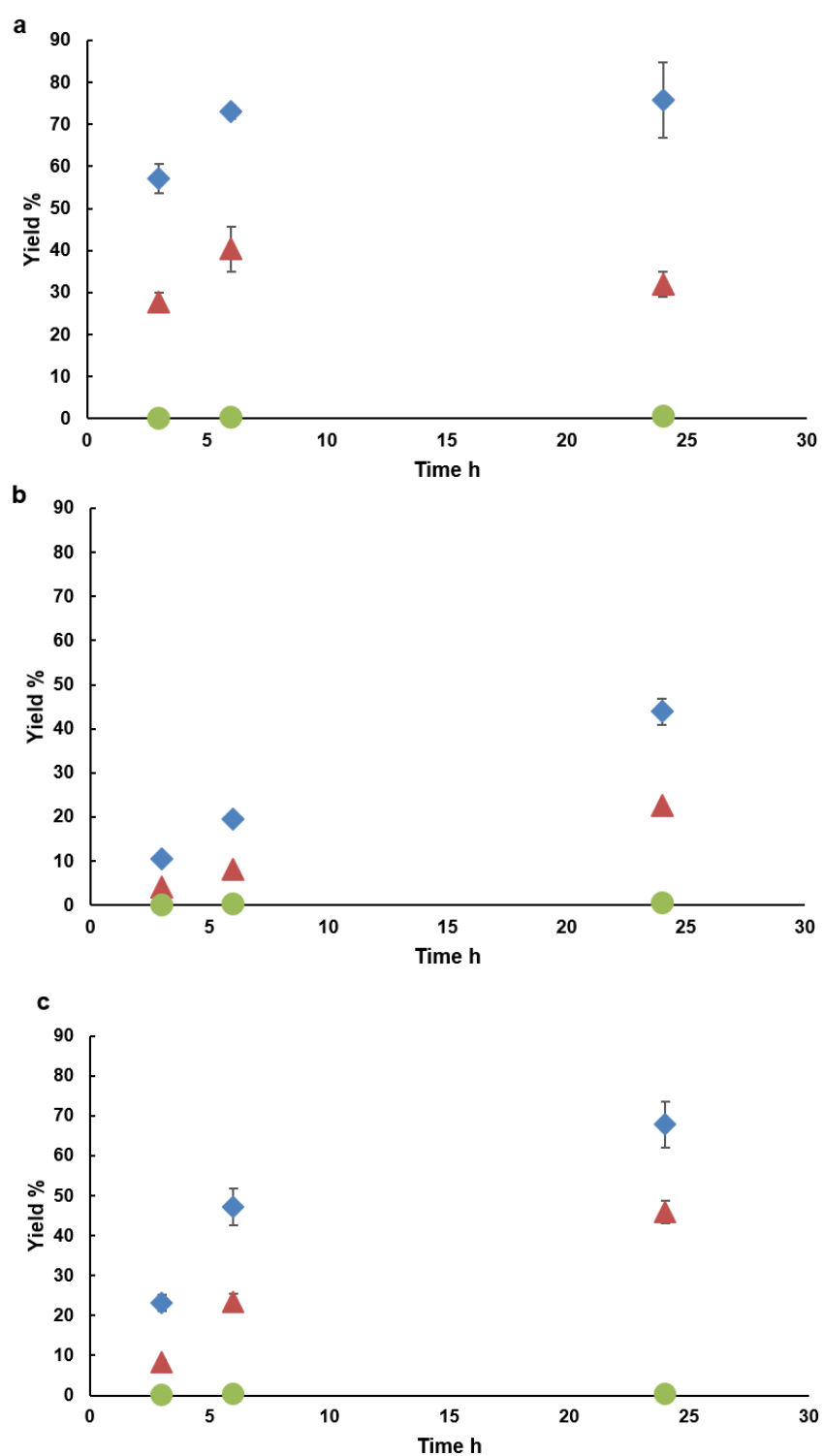

Figure 2. Hydrazone formation in vivo after the integration of CAR-SFP and FCS-ECH with LmrR_V15pAF_RMH in E.coli K12 MG1655 (RARE), using MMV at $24{ }^{\circ} \mathrm{C}$ with135 rpm for $24 \mathrm{~h}$. (a) hydrazone formation using $5 \mathrm{mM}$ 4b and $50 \mu \mathrm{M}$ 2. CAR-SFP/LmrR_V15pAF_RMH (blue) gave $57 \%, 73 \%$ and $76 \%$ after 3, 6, and $24 \mathrm{~h}$, respectively. Although CAR only (red) gave some background, LmrR_V15pAF_RMH only (green) did not yield any product. (b) 5 $\mathrm{mM} \mathrm{4a}$ and $50 \mu \mathrm{M} 2$ (c) $5 \mathrm{mM} 5$ and $50 \mu \mathrm{M}$ 2, the trend was not different from (a). FCS-ECH_LmrRV15pAF_RMH (blue) gave $23 \%, 47 \%$, and $68 \%$ of 3a after 3,6 , and $24 \mathrm{~h}$, respectively. The FCS-ECH only (red) gave a background of $46 \%$ after $24 \mathrm{~h}$. The results for LmrR_V15pAF_RMH (green) were the same for all substrates $(\mathbf{4 a}, \mathbf{b}, \mathbf{5})$. Error margins represent standard deviations from experiments performed in triplicate. 
Since significantly higher yields of product were observed when both CAR and LmrR_V15pAF_RMH were present, as compared to CAR alone, it can be concluded that the hydrazone product is formed predominantly from the combination of the heterologous biosynthesis of aldehyde and hydrazone formation catalyzed by the artificial enzyme.

Using whole cells expressing FCS-ECH / LmrR_V15pAF_RMH did give rise to good yields of hydrazone products based on exogenously added p-coumaric acid as starting material. The yield of hydrazone product was higher than obtained with the combination of CAR with LmrR_V15pAF_RMH. However, also in this case a non-neglible amount of product was formed in the control experiment without LmrR_V15pAF, showing the presence of significant background reactions.

\section{In vivo cascade of HMFO and LmrR_V15pAF_RMH in cells.} The cascade alcohol oxidation and hydrazone formation were first tested in vitro, using purified HMFO, in absence and presence of catalase, and LmrR_V15pAF_RMH with excellent yield, to establish if the $\mathrm{H}_{2} \mathrm{O}_{2}$ produced by HMFO would negatively affects the reaction (Figure.SI.9).Then we performed the in vivo oxidation and hydrazone formation cascade reactions using the whole cells expressing HMFO and LmrR_V15pAF_RMH. The reactions were set up with NBD-H (final concentration $50 \mu \mathrm{M}$ ) and benzyl alcohol (final concentration $5 \mathrm{mM}$ ) in cells for two hours in phosphate buffer $\mathrm{pH}$ 6.5. The cells expressing HMFO and LmrR_V15pAF_RMH gave the corresponding hydrazone product 3b with $81 \%$ yield after 2 hours. In contrast, the control experiment with cells only expressing HMFO gave only $6 \%$ yield, which is the background reaction. Using para-hydroxyl benzyl alcohol with $41 \%$ yield of the hydrazone product was obtained after 20 hours. Those results suggest the successful and efficient in vivo cascade pathway by the combination of a natural enzyme and an artificial enzyme. Since the HFMO LmrR_V15pAF_RMH gave such a large difference compared to background reaction, it ispossible to observe the difference in reactivity by visual inspection (Figure 3b).

\section{a}

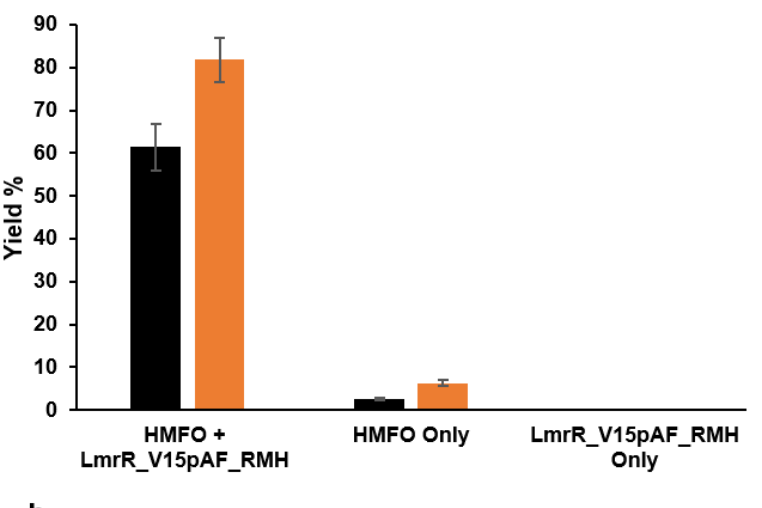

b

HMFO only

HMFO + LmrR_V15pAF RMH

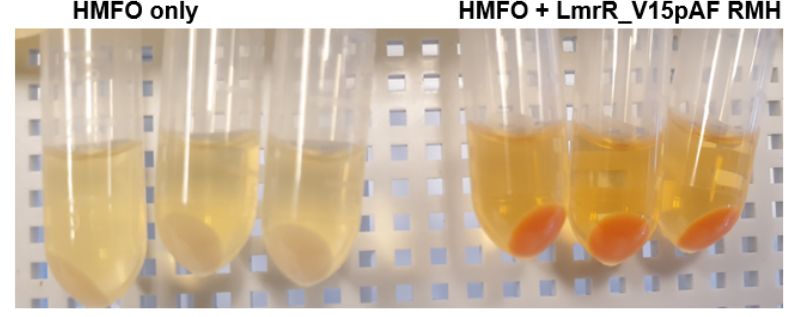

Figure 3 (a) The in vivo cascade hydrazone formation reaction in $2.5 \mathrm{~mL}$ cell culture with fresh phosphate buffer ( $\mathrm{pH} 6.5)$ by adding $50 \mu \mathrm{M} 2$ and $5 \mathrm{mM} \mathbf{6 b}$ at $24{ }^{\circ} \mathrm{C}$ with $135 \mathrm{rpm}$ for $1 \mathrm{~h}$ (black) to $2 \mathrm{~h}$ (orange). After $1 \mathrm{~h} 61 \%$ of $\mathbf{3 b}$ was detected and $81 \%$ after $2 \mathrm{~h}$. However, HMFO only and LmrR_V15pAF only yielded no product. Error margins represent standard deviations from experiments performed in triplicate. (b) Visual representation of hydrazone formation in phosphate buffer $\mathrm{pH} 6.5$, at $24{ }^{\circ} \mathrm{C}$ with $135 \mathrm{rpm}$ for $2 \mathrm{~h}$ with HMFO_LmrR_V15pAF_RMH (right) compared to HMFO only (left).

To confirm that the catalysis occurs in the cell and that the cells remain intact during and after catalysis a recycling experiment was performed. In the first round, we performed the cascade reaction by using $5 \mathrm{mM}$ of benzylalcohol and $50 \mathrm{uM}$ of NBD-H. The reaction was kept at $24{ }^{\circ} \mathrm{C} / 135 \mathrm{rpm}$ for 3 hours, resulting in $63 \%$ yield of the hydrazone product $\mathbf{3 b}$ (Figure SI.12). The cells were then washed with $50 \mathrm{mM} \mathrm{Kpi} \mathrm{buffer} \mathrm{at} \mathrm{pH} 6.5$ to remove all extracellular components and resuspended in reaction buffer. 5 mM p-hydroxybenzyl alcohol and 50 uM NBD-H were added. After reaction for 16 hours, the corresponding product (Figure SI.12) was obtained in $22 \%$ yield, accompanied by $\sim 10 \%$ residual product from the first round. This experiment confirms that the cells remain intact and that both the HMFO and the artificial enzyme remained inside the cells during the catalysis. 
The viability of the remaining cells after catalysis was tested by spinning them down and resuspending in $1 \mathrm{ml} \mathrm{LB}$, after which serial dilutions were plated on agar containing the required antibiotics. 336 colonies of HMFO/LmrR_V15pAF_RMH / E. coli RARE were still present on plates with cells diluted to $10^{-6}$ (Figure S17). This means that the reaction conditions, i.e. in presence of both the canonical and artificial enzymes, as well as the substrates and the hydrazone product, are well tolerated.

\section{Conclusion}

In conclusion, we have created in vivo biocatalytic cascades in $E$. coli that comprise a combination of natural and artificial enzymes. These results show that a heterologous biosynthetic pathway can be augmented with a new-to-nature catalytic reaction for the production of novel compounds. This work is a step towards the creation of a hybrid metabolism, which combines multiple natural and artificial enzymes in living cells to produce complex molecules from simple bio-based starting materials.

\section{Acknowledgements}

We wish to thank Prof M.W. Fraaije for the HMFO gene and the pBAD plasmid, Gecco Biotech for providing purified HMFO used in this study and R. Leveson-Gower for synthetic assistance. This work was supported by the European Research Council (ERC advanced grant 885396), the Ministry of Education, Culture and Science (Gravitation programme no. 024.001.035) and The Netherlands Organisation for Scientific Research (NWO, project 724.013.003)

Keywords: biocatalysis • artificial enzyme $\bullet$ in vivo cascade• biosynthetic pathways $\cdot$ hydrazone formation

[1] J. Nielsen, J. D. Keasling, Cell 2016, 164, 1185-1197.

[2] M. Jeschek, S. Panke, T. R. Ward, Trends Biotechnol. 2018, 36, 60-72.

[3] R. V. Maaskant, S. Chordia, G. Roelfes, ChemCatChem 2021, 1-8.

[4] S. Wu, Y. Zhou, D. Gerngross, M. Jeschek, T. R. Ward, Nat. Commun. 2019, 10, 1-10.

[5] G. Sirasani, L. Tong, E. P. Balskus, Angew. Chem. Int. Ed. 2014, 53, 7785-7788.

[6] S. Wallace, E. P. Balskus, Angew. Chem. Int. Ed. 2015, 54, 7106-7109.

[7] S. V. Sharma, X. Tong, C. Pubill-Ulldemolins, C. Cartmell, E. J. A. Bogosyan, E. J. Rackham, E. Marelli, R. B. Hamed, R. J. M. Goss, Nat. Commun. 2017, 8, 1-9.
[8] P. Destito, C. Vidal, F. López, J. L. Mascareñas, Chem. Eur. J. 2021, 27, 4789-4816.

[9] C. Adamson, M. Kanai, Org. Biomol. Chem. 2021, 19, 3745.

[10] J. G. Rebelein, T. R. Ward, Curr. Opin. Biotechnol. 2018, 53, 106-114.

[11] F. Schwizer, Y. Okamoto, T. Heinisch, Y. Gu, M. M. Pellizzoni, V. Lebrun, R. Reuter, V. Köhler, J. C. Lewis, T. R. Ward, Chem. Rev. 2018, 118, 142-231.

[12] S. Chordia, S. Narasimhan, A. Lucini Paioni, M. Baldus, G. Roelfes, Angew. Chem. Int. Ed. 2021, 2-10.

[13] W. Ghattas, V. Dubosclard, A. Wick, A. Bendelac, R. Guillot, R. Ricoux, J. P. Mahy, J. Am. Chem. Soc. 2018, 140, 8756-8762.

[14] A. R. Grimm, D. F. Sauer, T. Polen, L. Zhu, T. Hayashi, J. Okuda, U. Schwaneberg, ACS Catal. 2018, 8, 2611-2614.

[15] M. Jeschek, R. Reuter, T. Heinisch, C. Trindler, J. Klehr, S. Panke, T. R. Ward, Nature 2016, 537, 661-665.

[16] T. Heinisch, F. Schwizer, B. Garabedian, E. Csibra, M. Jeschek, J. Vallapurackal, V. B. Pinheiro, P. Marlière, S. Panke, T. R. Ward, Chem. Sci. 2018, 9, 5383-5388.

[17] M. Wittwer, U. Markel, J. Schiffels, J. Okuda, D. F. Sauer, U. Schwaneberg, Nat. Catal. 2021, 4, 814-827.

[18] M. A. S. Mertens, D. F. Sauer, U. Markel, J. Schiffels, J. Okuda, U. Schwaneberg, Catal. Sci. Technol. 2019, 9, 5572-5576.

[19] V. Köhler, Y. M. Wilson, M. Dürrenberger, D. Ghislieri, E. Churakova, T. Quinto, L. Knörr, D. Häussinger, F. Hollmann, N. J. Turner, T. R. Ward, Nat. Chem. 2013, 5, 93-99.

[20] J. Huang, Z. Liu, B. J. Bloomer, D. S. Clark, A. Mukhopadhyay, J. D. Keasling, J. F. Hartwig, Nat. Chem. 2021, DOI 10.1038/s41557-021-00801-3.

[21] I. Drienovská, C. Mayer, C. Dulson, G. Roelfes, Nat. Chem. 2018, 10, 946-952.

[22] I. Drienovská, G. Roelfes, Nat. Catal. 2020, 3, 193-202.

[23] P. K. Madoori, H. Agustiandari, A. J. M. Driessen, A. M. W. H. Thunnissen, EMBO J. 2009, 28, 156-166.

[24] L. Wang, A. Brock, P. G. Schultz, Science 2001, 292, 498500.

[25] R. A. Mehl, J. C. Anderson, S. W. Santoro, L. Wang, A. B. Martin, D. S. King, D. M. Horn, P. G. Schultz, J. Am. Chem. Soc. 2003, 125, 935-939.

[26] A. Erkkila“, I. Majander, P. M. Pihko, Chem. Rev. 2007, 107, 5416-5470.

[27] D. K. Kölmel, E. T. Kool, Chem. Rev. 2017, 117, 1035810376.

[28] E. H. Cordes, W. P. Jencks, J. Am. Chem. Soc. 1962, 84, 826-831.

[29] C. Mayer, C. Dulson, E. Reddem, A. M. W. H. Thunnissen, 
G. Roelfes, Angew. Chem. Int. Ed. 2019, 58, 2083-2087.

[30] R. B. Leveson-Gower, Z. Zhou, I. Drienovská, G. Roelfes, ACS Catal. 2021, 11, 6763-6770.

[31] Z. Zhou, G. Roelfes, Nat. Catal. 2020, 3, 289-294.

[32] M. Winkler, Curr. Opin. Chem. Biol. 2018, 43, 23-29.

[33] S. Wu, R. Snajdrova, J. C. Moore, K. Baldenius, U. T. Bornscheuer, Angew. Chem. Int. Ed. 2021, 60, 88-119.

[34] J. J. Dong, E. Fernández-Fueyo, F. Hollmann, C. E. Paul, M. Pesic, S. Schmidt, Y. Wang, S. Younes, W. Zhang, Angew. Chem. Int. Ed. 2018, 57, 9238-9261.

[35] A. M. Kunjapur, Y. Tarasova, K. L. J. Prather, J. Am. Chem. Soc. 2014, 136, 11644-11654.

[36] H. Stolterfoht, D. Schwendenwein, C. W. Sensen, F. Rudroff, M. Winkler, J. Biotechnol. 2017, 257, 222-232.

[37] P. Venkitasubramanian, L. Daniels, J. P. N. Rosazza, J. Biol. Chem. 2007, 282, 478-485.

[38] J. Ni, Y. Y. Gao, F. Tao, H. Y. Liu, P. Xu, Angew. Chem. Int. Ed. 2018, 57, 1214-1217.

[39] J. Overhage, H. Priefert, A. Steinbüchel, Appl. Environ. Microbiol. 1999, 65, 4837-4847.

[40] W. Yang, H. Tang, J. Ni, Q. Wu, D. Hua, F. Tao, P. Xu, PLoS One 2013, 8, e67339.

[41] J. P. Bennett, L. Bertin, B. Moulton, I. J. S. Fairlamb, A. M. Brzozowski, N. J. Walton, G. Grogan, Biochem. J. 2008, 414, 281-289.

[42] C. Martin, A. Ovalle Maqueo, H. J. Wijma, M. W. Fraaije, Biotechnol. Biofuels 2018, 11, 56.

[43] E. Romero, G. Gadda, Biomol. Concepts 2014, 5, 299-318. 
Supporting information

An In Vivo Biocatalytic Cascade featuring an artificial enzyme catalyzed new-to nature reaction.

Linda Ofori Atta, Zhi Zhou and Gerard Roelfes*

Table of Contents

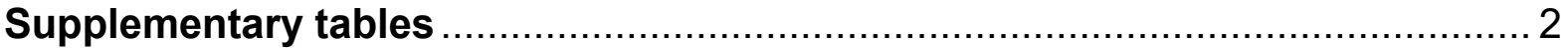

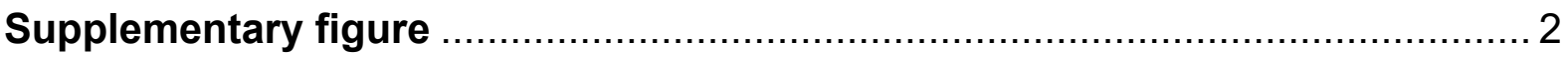

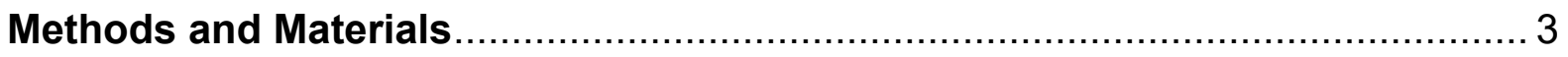

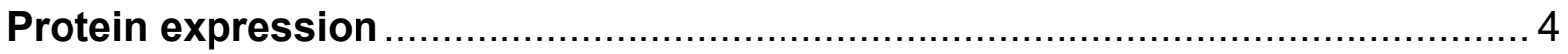

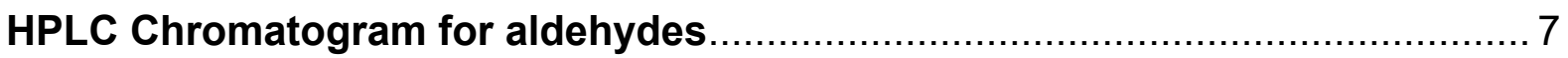

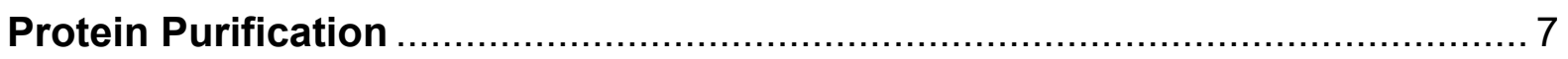

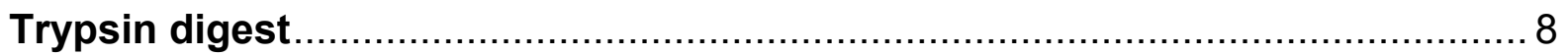

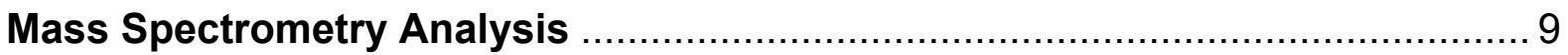

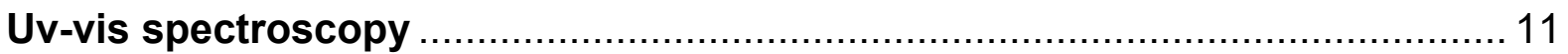

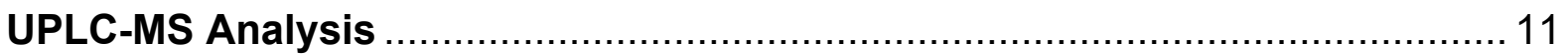

Hydrazone formation reaction in vivo / in vitro .......................................... 12

HPLC Chromatogram for hydrazones .................................................. 14

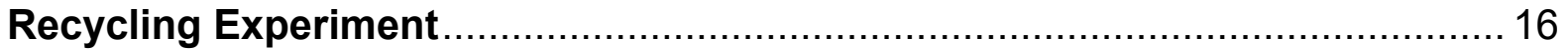

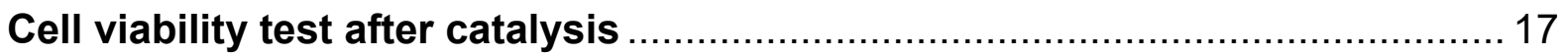

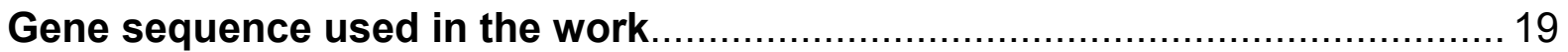

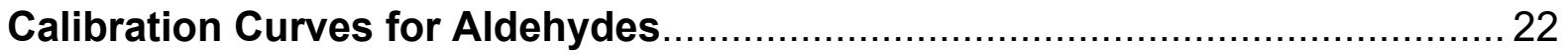

Chemical synthesis and calibration curves of hydrazone products $3 a$ and $3 \mathbf{b} .23$

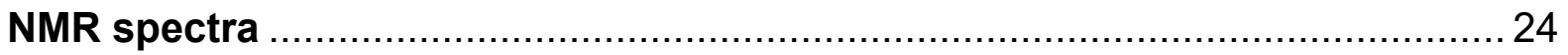

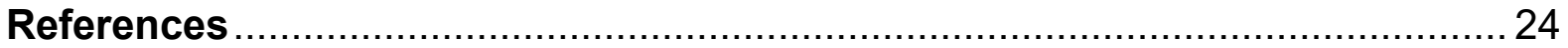




\section{Supplementary tables}

\begin{tabular}{|l|l|}
\hline E. coli Strains & Plasmids \\
\hline K-12 MG1655 (RARE) for expression & pETDuet-1 harbouring CAR-SFP \\
\hline NEB 5 alpha for cloning & pETDuet-1 harbouring FCS-ECH \\
\hline NEB 10 beta for cloning & pET28b+ harbouring LmrR-V15pAF_RMH \\
\hline & pBAD harbouring HMFO and LmrR-V15pAF_RMH \\
\hline & pDULE 2 harbouring pAF OTS \\
\hline
\end{tabular}

Table S1. Bacteria strains and plasmid used in this study.

\begin{tabular}{|l|l|l|}
\hline Protein Sample & Expression plasmid/OTS & $\boldsymbol{k}_{\text {cat }} / \boldsymbol{K}_{\mathbf{m}}$ (app) $\mathbf{M}^{-\mathbf{1}} / \mathbf{s}^{-1}$ \\
\hline LmrR_V15pAF_RMH & pEVOL_pAzF & 70 \\
\hline LmrR_V15pAF_RMH & pDULE 2 pAF & 54 \\
\hline
\end{tabular}

Table S2. Comparison of catalytic efficiency in the hydrazone formation reaction of $\mathbf{1 a}$ with $\mathbf{2}$ to form $\mathbf{3}$, by artificial enzymes produced using direct incorporation of pAF and indirect incorporation of PAF into LmrR_V15X RMH using the hydrazone formation reaction. ${ }^{[2]}$ Based on the relative activitiy it is concluded that the pAF incorporation efficiency is $\sim 80 \%$ when using the direct incorporation method. $\boldsymbol{k}_{\text {cat }} / K_{\mathrm{m}}(\mathbf{a p p}) \mathbf{M}^{-1} / \mathbf{S}^{-1}=$ catalytic efficiency Extinction Coefficient $(\varepsilon)=29,585 \mathrm{M}^{-1} \mathrm{~cm}^{-1}$.

\section{Supplementary figure}

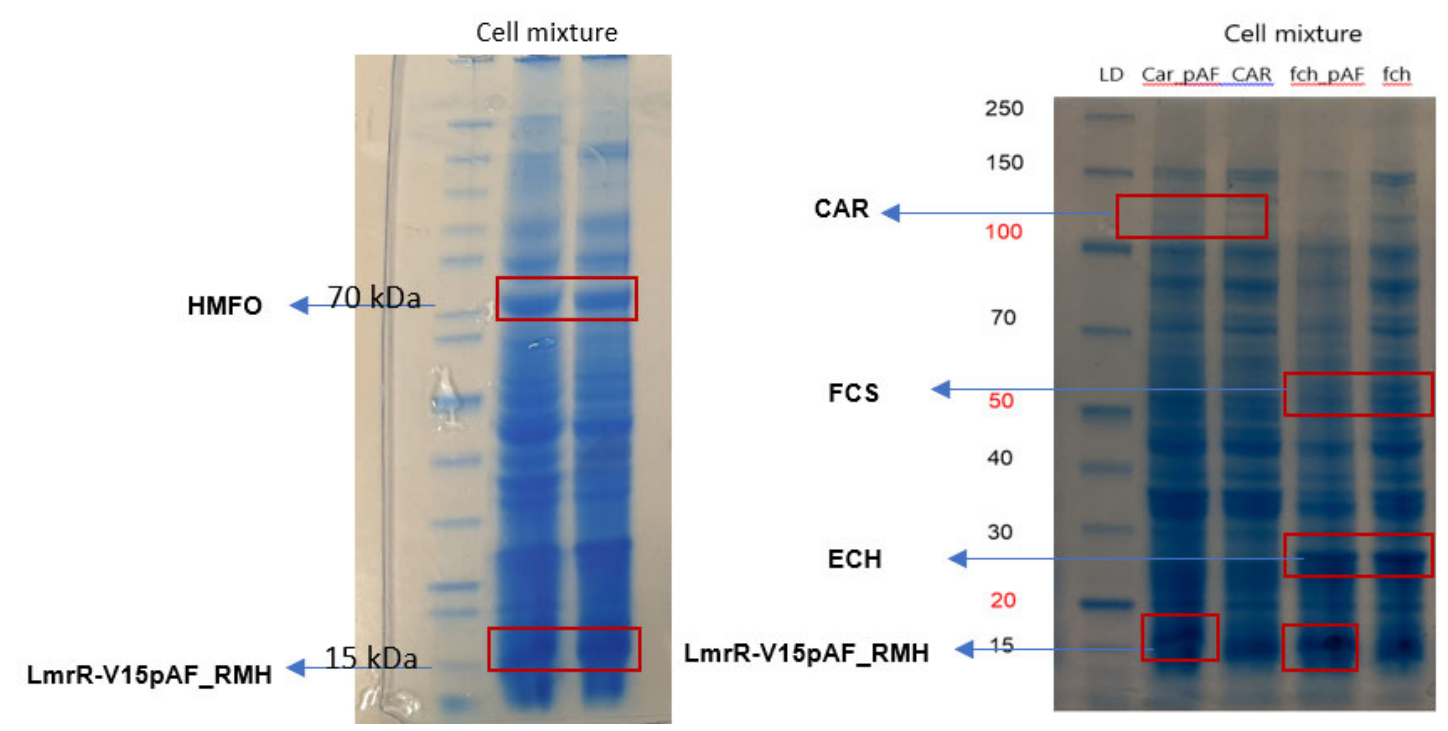

Figure S1. Expression of LmrR_V15pAF_RMH, CAR, FCS-ECH and HMFO after integration of the plasmids in E. coli RARE. CAR showed a ow expression, most likely caused by its large gene size of $4000 \mathrm{bp}$, making it difficult for RNA polymerase to synthesise the protein. 


\section{Methods and Materials}

Strains and Plasmids: E. coli strains and plasmids used in this study are listed in Table S1. Molecular biology techniques were performed using standard practices unless otherwise stated. Molecular cloning and vector propagation were performed in K-12 MG1655. The CAR gene from Nocardia iowensis and SFP gene from Bacillus subtilis, were synthesized and codon-optimized for expression in E. coli (GenScript USA). The pDULE plasmid harbouring pAF synthaseltRNA genes was purchased from addgene (pDule2-para-aminoPhe was a gift from Ryan Mehl, Addgene plasmid \#85503) ${ }^{[1]}$, the pBad plasmid harbouring HMFO was a gift from the Fraaije group (University of Groningen). Purified HMFO used in this study was a gift from Gecco Biotech (www.gecco-biotech.com).

Cell culture conditions: A standard recipe for Lysogenic Broth (LB) medium was used (10 g/L Bacto tryptone, $5 \mathrm{~g} / \mathrm{L}$ Yeast extract, $10 \mathrm{~g} / \mathrm{L} \mathrm{NaCl}$ and $15 \mathrm{~g} / \mathrm{L}$ Agar for LBAgar plates). MMV medium was prepared with the following recipe, $5.3 \mathrm{~g} / \mathrm{L} \mathrm{Na}_{2} \mathrm{HPO}_{4}$ $\times 12 \mathrm{H}_{2} \mathrm{O}, 1.4 \mathrm{~g} / \mathrm{L} \mathrm{KH}_{2} \mathrm{PO}_{4}, 0.2 \mathrm{~g} / \mathrm{L} \mathrm{MgSO}_{4} \times 7 \mathrm{H}_{2} \mathrm{O}, 1.0 \mathrm{~g} / \mathrm{L}\left(\mathrm{NH}_{4}\right)_{2} \mathrm{SO}_{4}$. The following components were sterile-filtered using $0.2 \mu \mathrm{m}$ filter before adding the medium: $1 \mathrm{ml} / \mathrm{L}$ vitamin solution $(1000 \times), 5 \mathrm{ml} / \mathrm{L}$ trace solution $(200 \times), 4 \mathrm{ml} / \mathrm{L}$ glucose $(50 \%)$. M9 medium was prepared using the following recipe, $56 \mathrm{~g} / \mathrm{L}$ of M9 salt (mix by Sigma) and a $0.2 \mu \mathrm{m}$ filter was used to filter the following component before addition $100 \mu \mathrm{l}$ of $1 \mathrm{M}$ $\mathrm{CaCl}_{2}, 2 \mathrm{~mL}$ of $1 \mathrm{M} \mathrm{MgSO}_{4}, 8 \mathrm{~mL}$ of $50 \%$ glucose solution, $5 \mathrm{~mL}$ of $4 \%$ vitamin B1 (thiamine), $50 \mathrm{~mL}$ of $20 \%$ Cas-amino acids. 


\section{Protein expression}

For experiments testing the direct incorporation of pAF into LmrRV15TAG, pDULE vector harboring pAF synthaseltRNA and $p E T 17 b+$ vector harboring LmrRV15TAG were co-transformed into E. coli k-12 MG1655 (RARE). Culture media were supplemented with $100 \mu \mathrm{g} / \mathrm{L}$ ampicillin and $10 \mu \mathrm{g} / \mathrm{L}$ streptomycin to provide selective pressure for plasmid maintenance. Except for cultures grown in LB medium, overnight pre-cultures were pelleted and re-suspended in either M9 medium or MMV medium. This step was done to ensure that nutrient rich LB medium was removed. Experimental cultures were then initiated by adding $2 \%(\mathrm{v} / \mathrm{v})$ of re-suspended pre-cultures into 50 $\mathrm{ml}$ either M9 or MMV medium while $1 \%(\mathrm{v} / \mathrm{v})$ pre-culture was added to LB medium. Gene expression was then induced with $1 \mathrm{M}$ isopropyl- $\beta$-d-1-thiogalactopyranoside (IPTG) (1 mM final concentration) and $20 \%$ L- arabinose (0.02 \% final concentration) between an $\mathrm{OD}_{600}$ of 0.4 and 1. During induction, $1 \mathrm{M} \mathrm{pAF}$ ( $1 \mathrm{mM}$ final concentration) was added in solid form, incubated at $24^{\circ} \mathrm{C}$, and agitated at $135 \mathrm{rpm}$ for $48 \mathrm{hrs}$. These experiments were performed in $250 \mathrm{ml}$ baffled Erlenmeyer flasks for better aeration.

\begin{tabular}{|l|l|l|l|l|}
\hline Media/Sample & $\begin{array}{l}\text { Strain } \\
\text { E.Coli K-12 } \\
\text { MG1655 }\end{array}$ & pAF & Temperature & Time \\
\hline LB & RARE & $1 \mathrm{mM}$ & $30^{\circ} \mathrm{C}$ & $16 \mathrm{~h}$ \\
\hline LB control & RARE & No pAF added & $30^{\circ} \mathrm{C}$ & $16 \mathrm{~h}$ \\
\hline M9 & RARE & $1 \mathrm{mM}$ & $30^{\circ} \mathrm{C}$ & $16 \mathrm{~h}$ \\
\hline M9 control & RARE & No pAF added & $30^{\circ} \mathrm{C}$ & $16 \mathrm{~h}$ \\
\hline MMV & RARE & $1 \mathrm{mM}$ & $24{ }^{\circ} \mathrm{C}$ & 48 hours \\
\hline MMV control & RARE & No pAF added & $24^{\circ} \mathrm{C}$ & 48 hours \\
\hline
\end{tabular}

Table S3. Expression conditions for pAF incorporation into LmrR_V15X_RMH. 


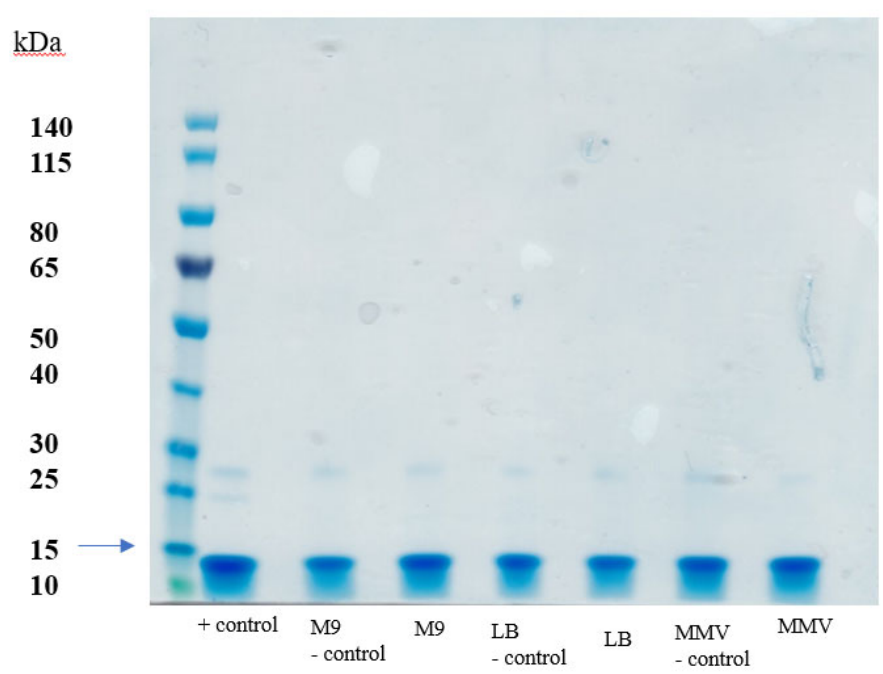

Figure S2. SDS-PAGE analysis of pAF incorporation into LmrR-V15X_RMH. Controls without PAF added do yield protein, which is caused by misincorporation of canonical amino acids due to the promiscuity of the orthogonal translation system.

\section{Metabolites analysis:}

For aromatic aldehydes synthesis, experiments were performed in 250/500 ml Erlenmeyer flasks (Sigma), using $50 \mathrm{~mL}$ MMV, supplemented with $100 \mu \mathrm{g}$ ampicillin. Experimental cultures were initiated by adding $1 \%(\mathrm{v} / \mathrm{v})$ inoculum volumes of overnight pre-culture to $50 \mathrm{ml}$ volume of $\mathrm{MMV}$, incubated at $37^{\circ} \mathrm{C}$, and agitated at $135 \mathrm{rpm}$.

Expression was induced with $1 \mathrm{mM}$ isopropyl- $\beta-\mathrm{d}-1$-thiogalactopyranoside (IPTG) or $0.02 \%$ arabinose between optical density $600\left(\mathrm{OD}_{600}\right)$ of $0.8-1.0$. The cultures were incubated at $24{ }^{\circ} \mathrm{C}$ and $135 \mathrm{rpm}$ for $16 \mathrm{~h}$. Cultures were spun down at $4000 \mathrm{rpm}$ for 10 mins, pellets were resuspended with fresh MMV and $5 \mathrm{mM}$ of substrate was added at $24{ }^{\circ} \mathrm{C}$ and $135 \mathrm{rpm}$ for $16 \mathrm{~h}$. For benzaldehyde producing cultures, samples were spun down and filtered with a $0.2 \mu \mathrm{M}$ filter before HPLC analysis. In cultures producing para hydroxy benzaldehyde, the products were extracted with ethyl acetate.

HPLC analysis using an Agilent series instrument equipped with a diode array detector was used to detect products at wavelengths of $247 \mathrm{~nm}$ and $281 \mathrm{~nm}$ for benzaldehyde and para-hydroxy benzaldehyde, respectively. A kinetex-C18 column was used and the gradient method used the following solvents:

(A1) acetonitrile $+0.1 \%$ trifluoroacetic acid (TFA); (B1) water $+0.1 \%$ TFA. The gradient started with $5 \%$ Solvent $A 1$ and $95 \%$ Solvent B1 with a flow rate of 1.0 $\mathrm{mL} / \mathrm{min}$, and all compounds of interest eluted within 30 mins. The column temperature was maintained at $25^{\circ} \mathrm{C}$. 


\begin{tabular}{|l|l|l|l|l|}
\hline Gene & Strain E.Coli K-12 MG1655 & Substrate (5 mM ) & Temperature & Time (hours) \\
\hline CAR_SFP & RARE & Benzoic Acid & $24^{\circ} \mathrm{C}$ & $16 \mathrm{~h} / 48 \mathrm{~h}$ \\
\hline CAR_SFP & RARE & $\begin{array}{l}4 \text { hydroxy-benzoic } \\
\text { Acid }\end{array}$ & $24{ }^{\circ} \mathrm{C}$ & $16 \mathrm{~h} / 48 \mathrm{~h}$ \\
\hline FCS-ECH & RARE & 4 -coumaric acid & $24^{\circ} \mathrm{C}$ & $16 \mathrm{~h} / 48 \mathrm{~h}$ \\
\hline HMFO & RARE & benzyl alcohol & $24^{\circ} \mathrm{C}$ & $16 \mathrm{~h} / 48 \mathrm{~h}$ \\
\hline HMFO & RARE & $\begin{array}{l}4 \text { hydroxy-benzyl } \\
\text { alcohol }\end{array}$ & $24^{\circ} \mathrm{C}$ & $16 \mathrm{~h} / 48 \mathrm{~h}$ \\
\hline
\end{tabular}

Table S4. Expression conditions for in vivo aldehydes production using E.coli K12 MG1655 (RARE).

a

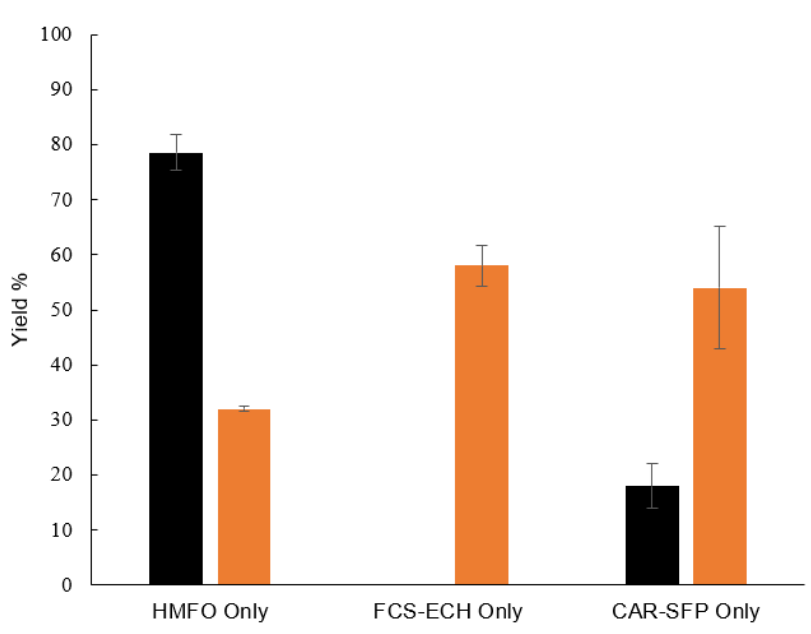

b

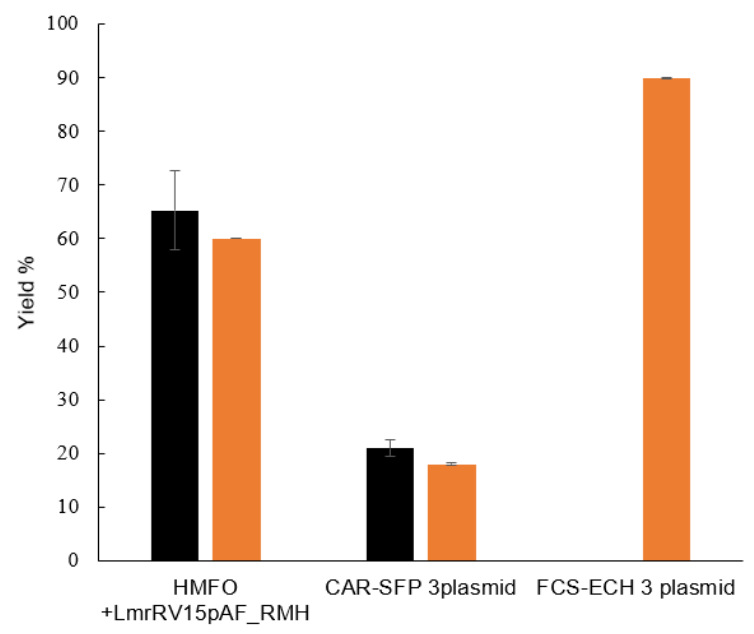

Figure S3. In vivo production of aldehydes before and after the integration of natural enzymes with LmrR-V15pAF_RMH in E.coli K12 MG1655 (RARE). (a) Benzaldehyde (black) was produced by HMFO and CAR-SFP and para hydroxy benzaldehyde (orange) was produced by HMFO, CAR-SFP and FCS- ECH. Expression was done at $24^{\circ} \mathrm{C} / 135 \mathrm{rpm}$ for $24 \mathrm{~h}$. (b) Aldehydes production using 3 plasmid system (CAR-SFP, FCS=ECH) and 2 plasmid system (HMFO), after the integration of natural enzymes with artificial enzyme (LmrR-V15pAF_RMH). Expression was done at $24^{\circ} \mathrm{C} / 135 \mathrm{rpm}$ for $48 \mathrm{~h}$. 


\section{HPLC Chromatogram for aldehydes}

a

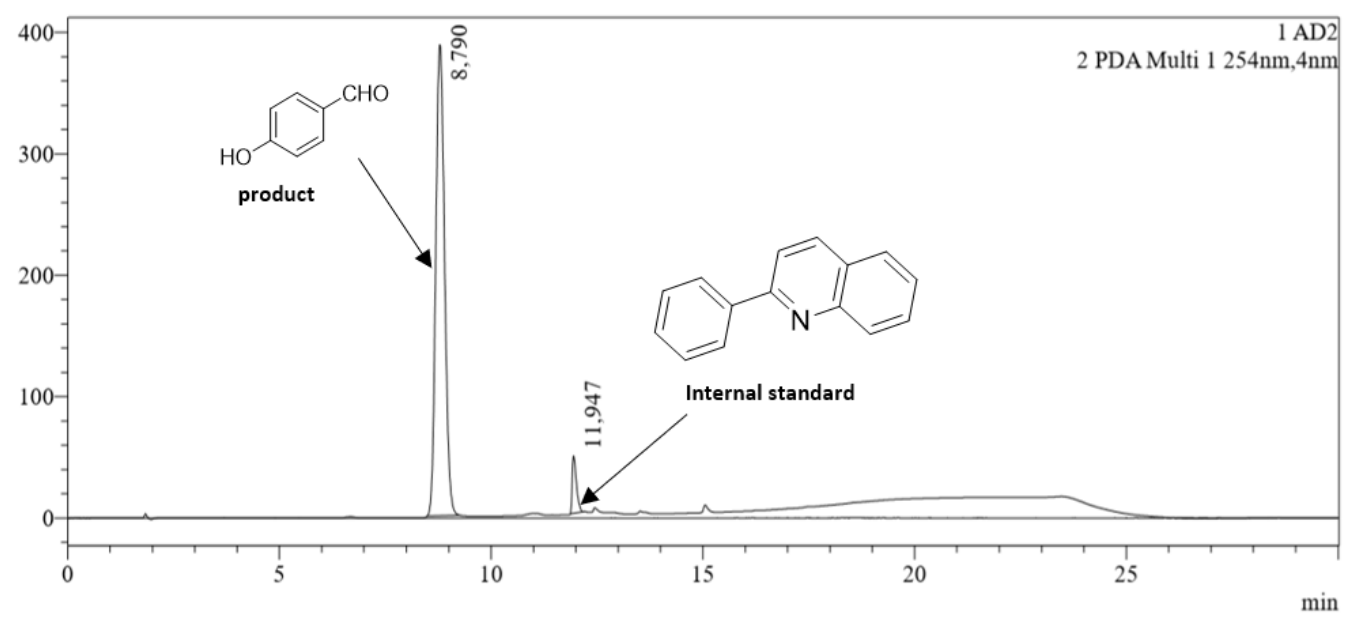

b

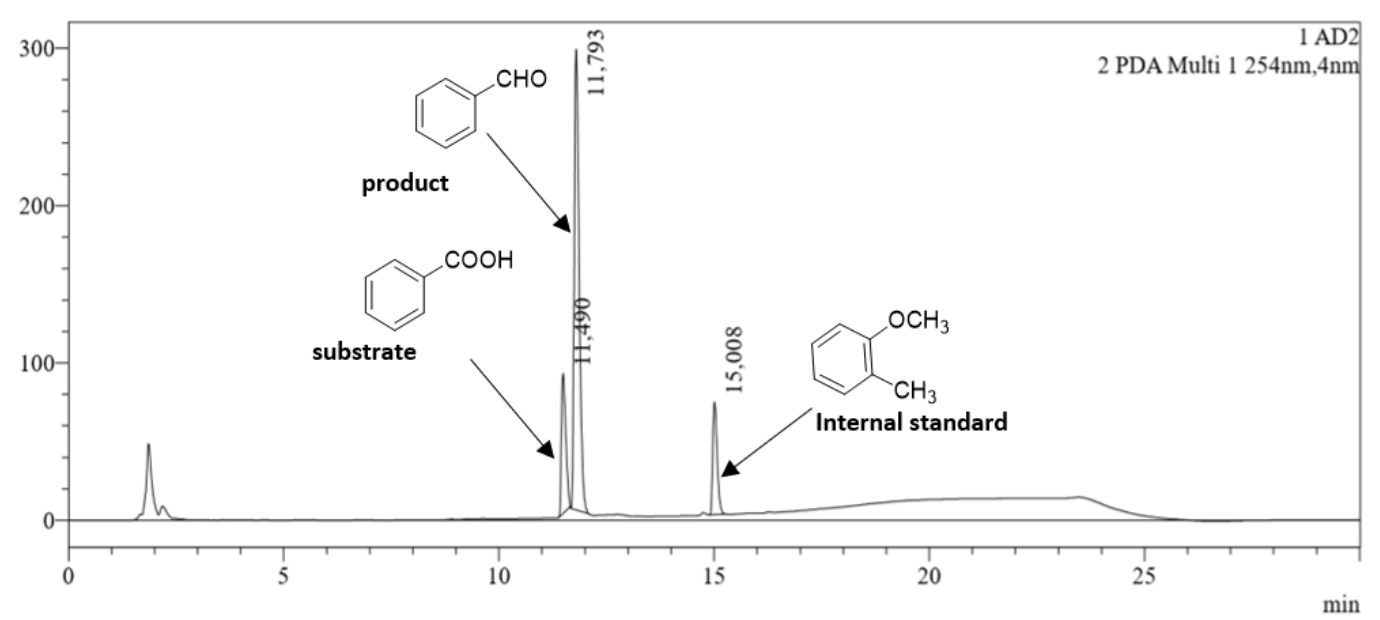

Figure S4. HPLC chromatogram of aldehydes biosynthesised in E.coli K12 MG1655 (RARE). (a) p-OH benzaldehyde product 3a peak detected at 8,7 min while the internal standard 2-Phenylquinoline had a retention time around 11,9 min. (b) Benzaldehyde product $\mathbf{3 b}$ peak detected at 11,7 min while the benzoic acid substrate peak had a retention time of 11,4 mins. Here 2-methylanisole was used as an internal standard.

\section{Protein Purification:}

Sodium phosphate buffer (in milliQ water) was prepared with the following recipe: 50 $\mathrm{mM}$ of $\mathrm{Na}_{2} \mathrm{HPO}_{4} ; 12 \mathrm{H}_{2} \mathrm{O}, 150 \mathrm{mM}$ of $\mathrm{NaCl}$ at pH 8. The buffer was filtered with $0.2 \mu \mathrm{m}$ sterile filters. After 48 hours of expression at $24{ }^{\circ} \mathrm{C}$, cells were harvested by centrifugation (6,000 rpm., JA10, Beckman) for $20 \mathrm{~min}$, at $4{ }^{\circ} \mathrm{C}$. The cell pellet was resuspended in $20 \mathrm{ml}$ Sodium phosphate buffer and half a tablet "mini complete protease inhibitor cocktail" (Roche) was added. The solutions were sonicated on ice (8 min, pulse of $10 \mathrm{~s}$ ON and $15 \mathrm{~s}$ OFF, amplitude of $70 \%$ ). 
The lysed cells were incubated on ice with $0.1 \mathrm{mg} / \mathrm{ml}$ of DNase I and $10 \mathrm{mM}$ of $\mathrm{MgCl}_{2}$ for $30 \mathrm{~min}$. The mixture was spun down with rotor JA17(Beckman), 12,000 rpm, for 60 $\min , 4{ }^{\circ} \mathrm{C}$. The supernatant was filtered using a $0.45 \mu \mathrm{m}$ filter and was loaded on a Strep-Tactin column (Strep-Tactin Superflow high capacity), incubated for 60 min at 4 ${ }^{\circ} \mathrm{C}$, and the protein was purified according to the manufacturer's guidelines. Eluents were collected and dialysed against the reaction buffer $\left(50 \mathrm{mM} \mathrm{NaH}_{2} \mathrm{PO}_{4}, 150 \mathrm{mM}\right.$ $\mathrm{NaCl}, \mathrm{pH} 7.4$, two times against $1 \mathrm{~L}$ ). The concentration of the protein was determined by using the calculated extinction coefficient for LmrR, corrected for the absorbance of $\operatorname{pAF}\left(\varepsilon 280=1,333 \mathrm{M}^{-1} \mathrm{~cm}^{-1}\right)$.

\section{Trypsin digest}

Protein for trypsin digest was initially purified by Streptag affinity chromatography and desalted with dialysis. $4 \mu \mathrm{l}$ of the LmrRv15pAF_RMH protein solution was diluted to $20 \mu \mathrm{l}$ using a $100 \mathrm{mM}$ ammonium bicarbonate (ABC) solution. $20 \mu \mathrm{l}$ of a 10 $\mathrm{ng} / \mu \mathrm{l}$ trypsin solution in $100 \mathrm{mM} \mathrm{ABC}$ was added and incubated overnight at $37^{\circ} \mathrm{C}$. The digestion mixture was acidified by adding $4 \mu$ formic acid and desalted using a C18 stage tip. The eluted peptides were dried in a speedvac and dissolved in $20 \mu \mathrm{l}$ $0.1 \%$ formic acid and injected into the nanoLC system for LCMSMS analysis. Resulting data were analysed using PeaksX software (BioSolutions Inc., Waterloo) Ontario, Canada).
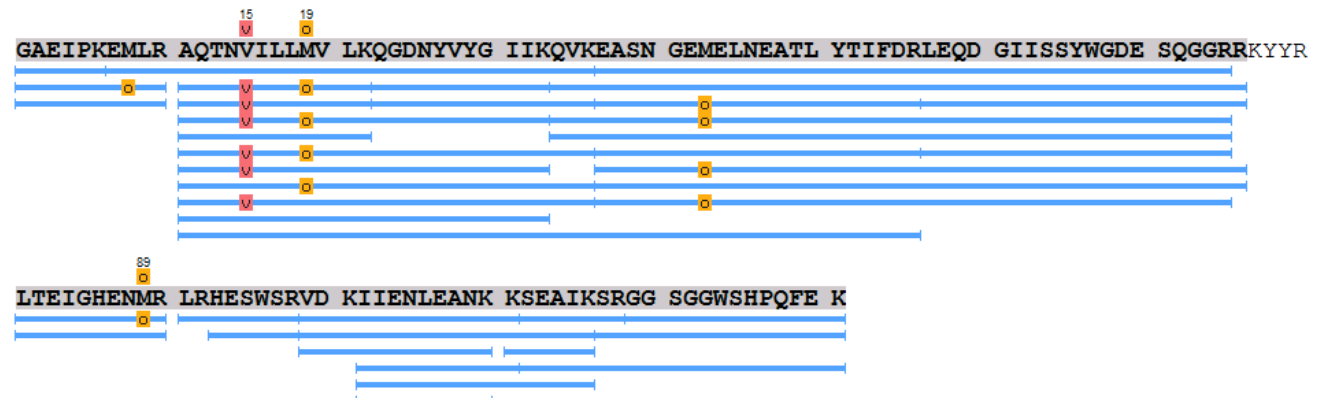

Figure S5. Trypsin digest of LmrR-V15pAF_RMH expressed in MMV. 


\section{Mass Spectrometry Analysis}

The mass spectrometry results showed protein expressed in M9 media had phenylalanine misincorporated and protein expressed in LB had a mixture of pAF and phenylalanine incorporated. Protein produced using the MMV media had pAF incorporated with high selectivity..

\section{Expected Mass $=15126 ;$ Observed Mass $=15126.64000$}

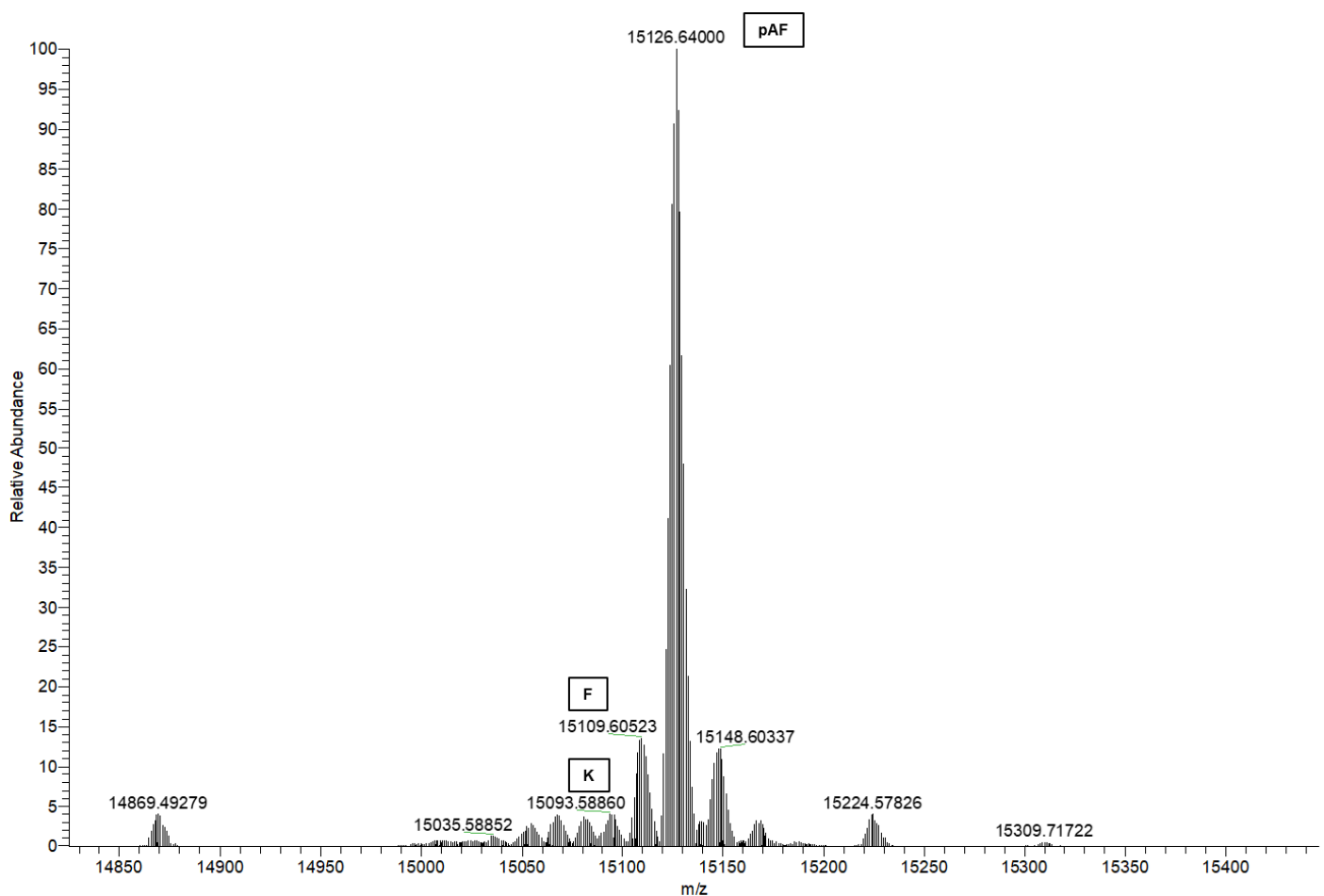

Figure S6. Mass of LmrR-V15pAF_RMH expressed in MMV. After deconvolution, an observed mass of 15126.64, which corresponds to the expected mass of LmrR-V15pAF_RMH, was detected. Although expression in this media gives some misincorporation of phenylalanine $(\mathrm{F})$ and Lysine $(\mathrm{K})$, around $80 \%$ of incorporated amino acid was pAF (unnatural amino acid). 


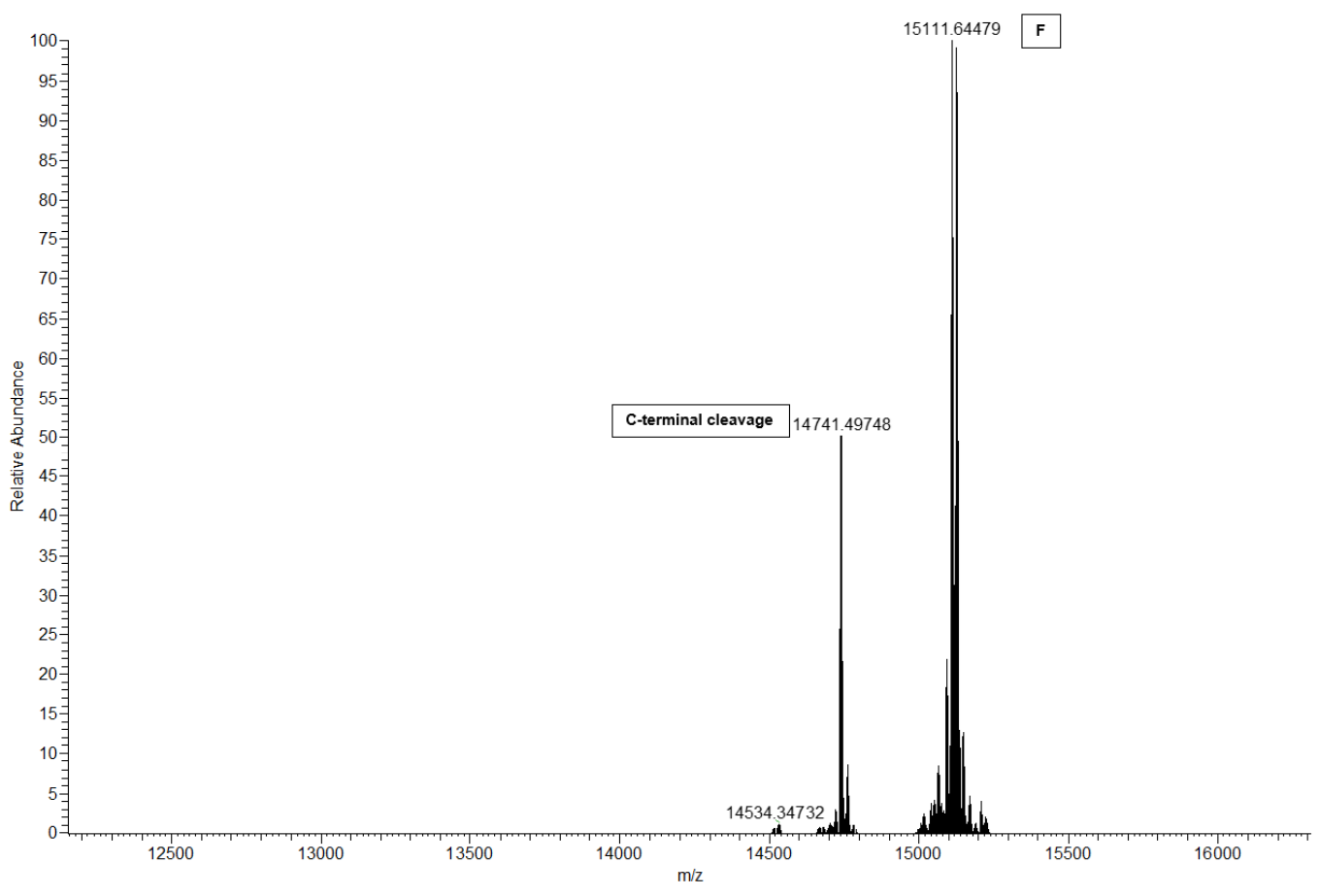

Figure S7. Mass of LmrR-V15X_RMH expressed in M9 media. After deconvolution, an observed mass of 15111.64 which corresponds to the expected mass of LmrR-V15X_RMH when phenylalanine $(F)$ is incorporation instead of pAF. A mass corresponding to the cleavage of 4 amino acids from the C-terminal was also detected.

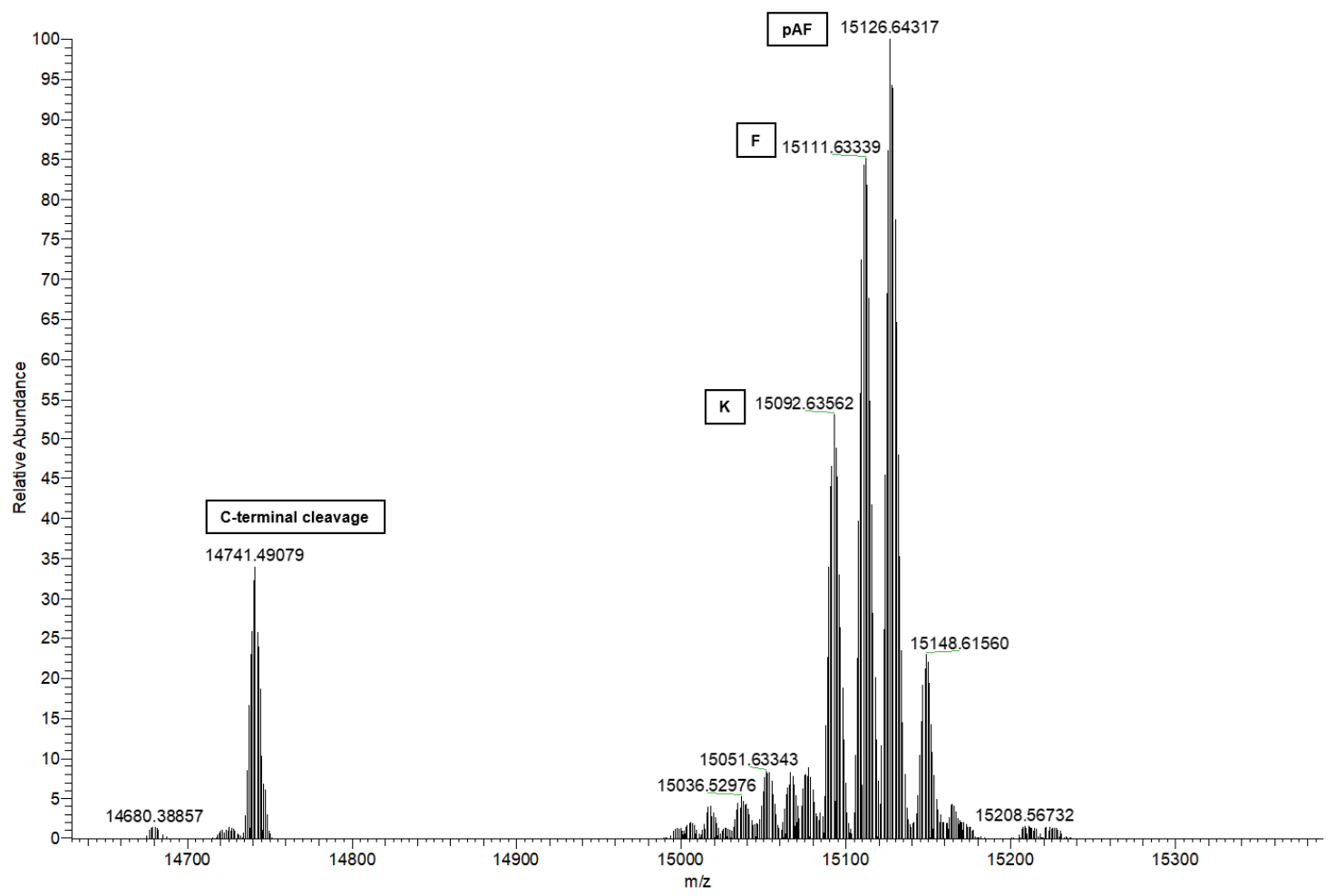

Figure S8. Mass of LmrR-V15X_RMH expressed in LB media. Although after deconvolution an observed mass of 15126.64 which corresponds to the expected mass of LmrR-V15pAF_RMH was detected, there was also a lot of misincorporation of phenylalanine (F) and Lysine (K). The presence of $\mathrm{C}$-terminal cleavage was also detected as it was with M9. 


\section{Uv-vis spectroscopy}

After hydrazone formation reaction with para hydroxy benzaldehyde (1a) and NBDH (2), the absorbance of $1 \mathrm{ml}$ of product 3a solution was measured.

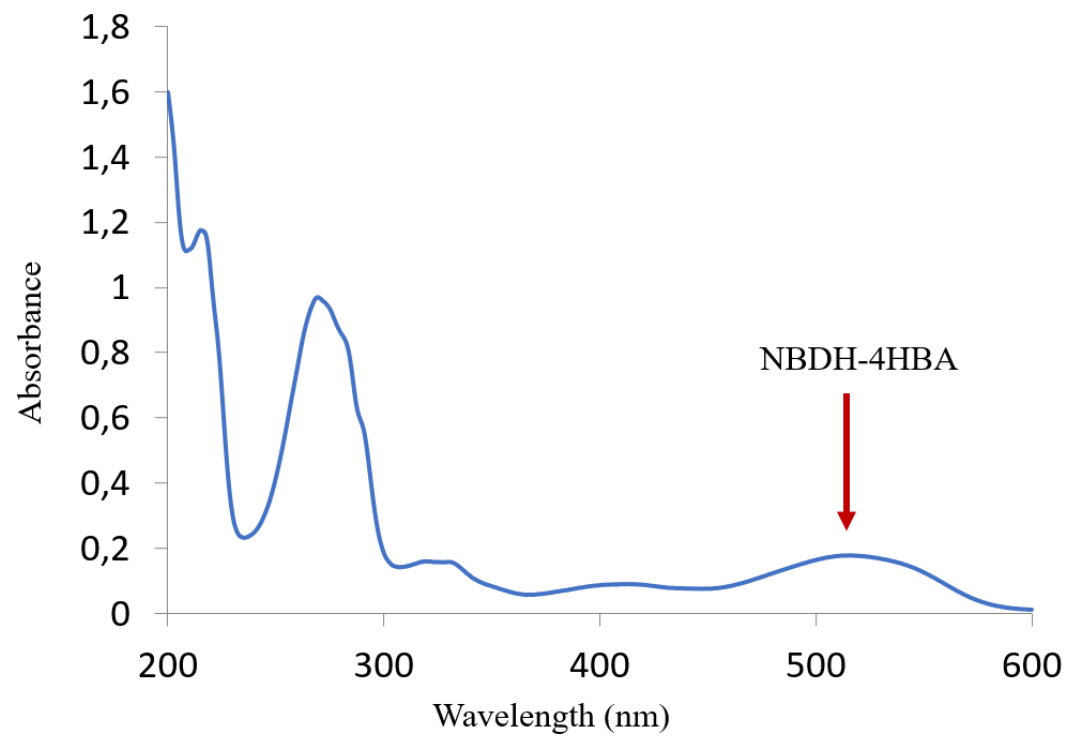

Figure S9. Uv-Vis spectra analysis of product 3a. The characteristic absorption band of the NBDH-4HBA product (3a) was detected at a wavelength of $520 \mathrm{~nm}$.

\section{UPLC-MS Analysis}

Reactions samples were pelleted by centrifugation, and aqueous supernatant was collected and filtered with $2 \mu \mathrm{m}$ filter for UPLC-MS analysis using ACQUITY UPLC $®$ HSS T3 $1.8 \mu \mathrm{m}$. The solvents used were (A) acetonitrile $+0.1 \%$ formic acid (TFA); (B) water $+0.1 \%$ formic acid Run for 20 mins.

a

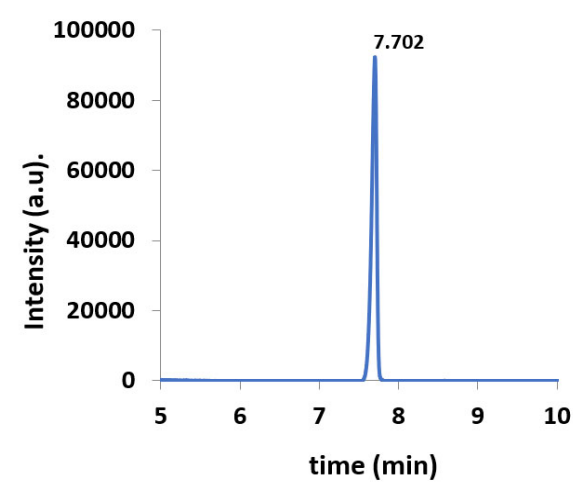

b

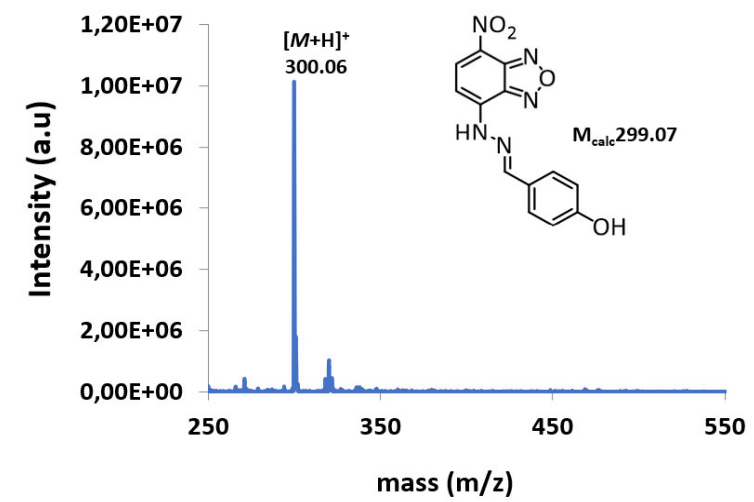

Figure S10. UPLC-MS analysis to confirm the mass of hydrazone product. (a) single peak at time 7.7 min corresponded to the retention time of hydrazone on the UPLC. (b)The mass of the calculated hydrazone was confirmed. 


\section{Hydrazone formation reaction in vivo / in vitro}

Hydrazone formation reaction in vivo: Experimental culture cells were grown in $50 \mathrm{ml}$ MMV supplemented with antibiotics at $37^{\circ} \mathrm{C}, 135 \mathrm{rpm}$ until $\mathrm{OD}_{600}$ of $0.4-0.7$. Gene expression was induced with $1 \mathrm{mM}$ isopropyl- $\beta$-d-1-thiogalactopyranoside (IPTG) and $0.02 \% \mathrm{~L}$ - arabinose. During induction $1 \mathrm{mM}$ pAF was added in solid form, incubated at $24{ }^{\circ} \mathrm{C}$, and agitated at $135 \mathrm{rpm}$ for $48 \mathrm{hrs}$. Cultures were pelleted and resuspended in newly prepared MMV, to wash away non-incorporated UAA, or in reaction buffer. In the $2.5 \mathrm{~mL}$ cell culture with newly prepared MMV medium was added $25 \mu \mathrm{L} \mathrm{NBDH}$ (2) (5 mM in DMF) solution (final concentration $50 \mu \mathrm{M}$ ) and $62.5 \mu \mathrm{L}$ carbonic acid or aldehyde (200 mM in DMF) solution (final concentration $5 \mathrm{mM}$ ). Then the mixture was put into the $24{ }^{\circ} \mathrm{C}$ shaker at $135 \mathrm{rpm}$ for 1 to 24 hours. The formation of the product was determined by HPLC. Take $500 \mu \mathrm{L}$ reaction culture into $2 \mathrm{ml}$ Eppendorf, added $50 \mu \mathrm{L}$ IS (1 mM Fluorescein in DMF), then extracted with $1 \mathrm{ml}$ ethyl acetate, after the evaporation, the residue was resuspended in $1200 \mu \mathrm{L} \mathrm{CH} \mathrm{CH}_{3} \mathrm{CN} .200 \mu \mathrm{L}$ of this solution was placed into HPLC vial and the sample was analyzed by HPLC.

Hydrazone formation reaction in vitro was set-up as follows: To $280 \mu \mathrm{L}$ of $5 \mu \mathrm{M}$ HMFO and $4 \mu \mathrm{M}$ LmrR_V15pAF_RMH in freshly prepared phosphate buffer pH 6.5 was added $10 \mu \mathrm{L}$ NBDH (1.5 mM in DMF) solution (final concentration $50 \mu \mathrm{M}$ ) and $10 \mu \mathrm{L}$ carbonic acid (150 $\mathrm{mM}$ in DMF) solution (final concentration $5 \mathrm{mM}$ ). Then the mixture was put into the $24{ }^{\circ} \mathrm{C}$ shaker at $135 \mathrm{rpm}$ for 1 to 3 hours. The yield of the products was determined by HPLC. To the reaction mixture was added $50 \mu \mathrm{L}$ internal standard (1 mM Fluorescein in DMF), then it was extracted with $200 \mu \mathrm{L}$ ethyl acetate. After the evaporation, the residue was resuspended in $240 \mu \mathrm{L} \mathrm{CH}{ }_{3} \mathrm{CN}$. $200 \mu \mathrm{L}$ of this solution was placed into HPLC vial and the sample was analyzed by HPLC. 


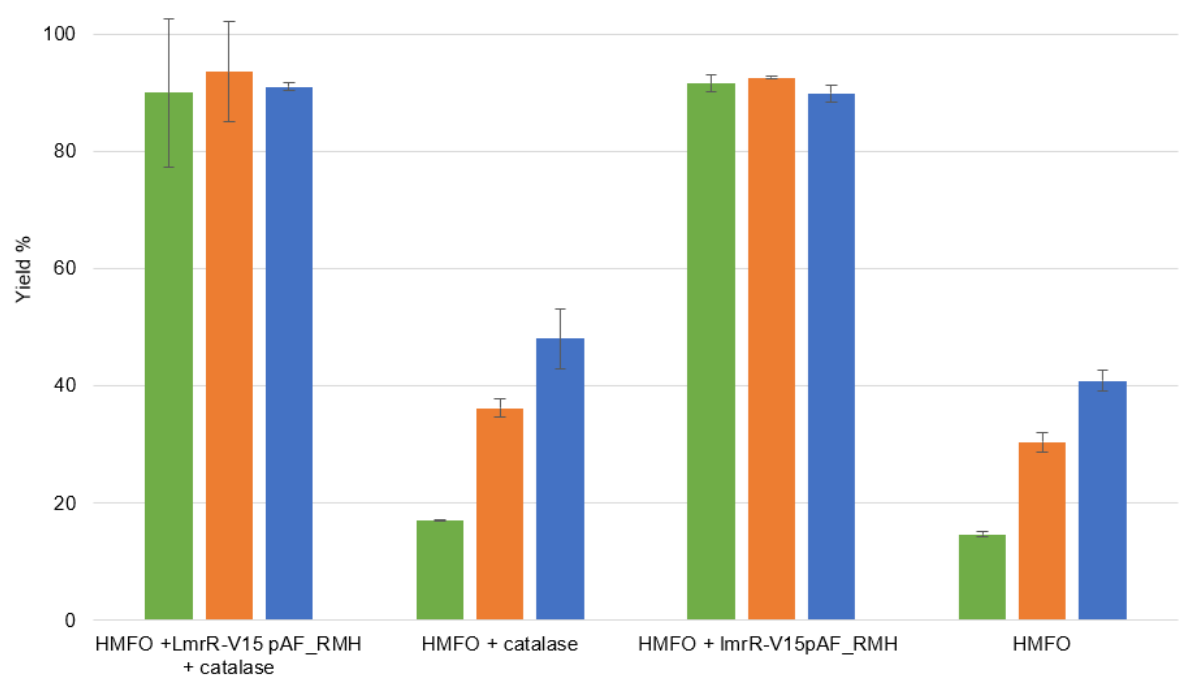

Figure S11. Hydrazone formation by the combination of HMFO and LmrR_V15pAF_RMH in vitro.

The in vitro reaction using the combination of HMFO and LmrR-V15pAF_RMH gave $85 \%$ yield after $1 \mathrm{~h}$ (green). Monitoring at 2 $\mathrm{h}$ (orange) and $3 \mathrm{~h}$ (blue) didn't give rise to further increased product formation. The control experiments with catalase showed that $\mathrm{H}_{2} \mathrm{O}_{2}$ has no effect on the cascade reactions.<smiles>NNc1ccc([N+](=O)[O-])c2nonc12</smiles>

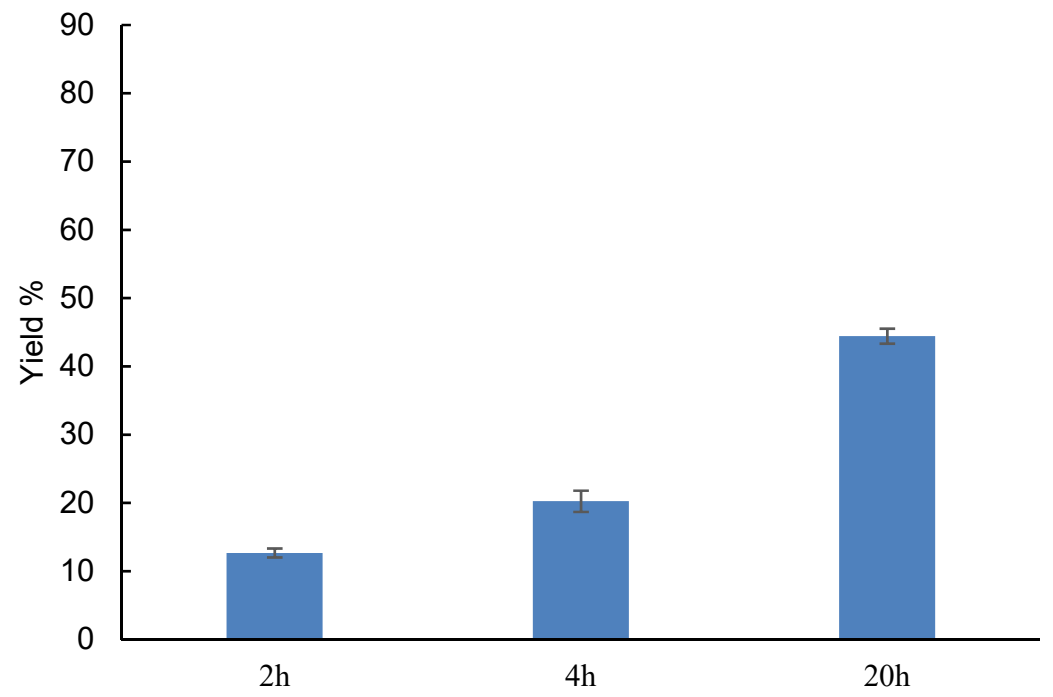

Figure S12. Hydrazone formation reaction of $\mathbf{1 a}$ and $\mathbf{2}$ by integration of HMFO and LmrR_V15pAF_RMH in vivo. The reaction was followed during $20 \mathrm{~h}$ with a final yield of $50 \%$ of product $3 \mathrm{a}$ formed. 


\section{HPLC Chromatogram for hydrazones}
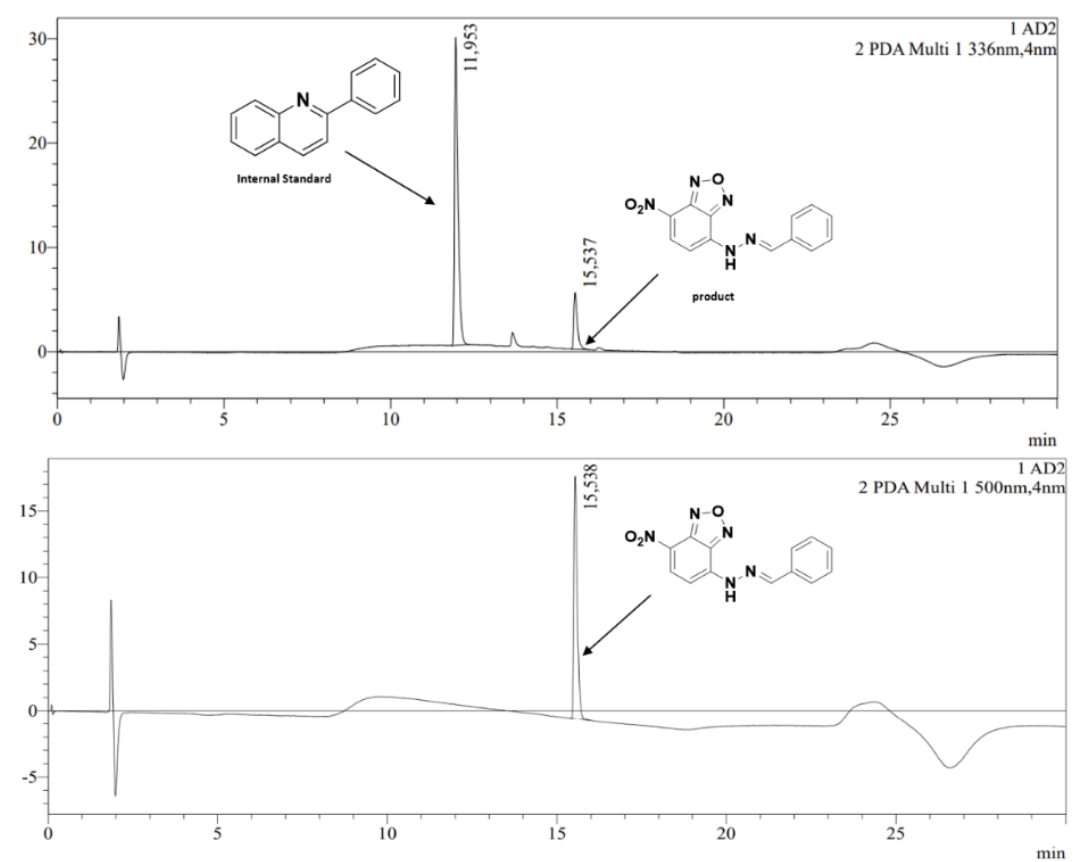

Figure S13. HPLC chromatogram of Hydrazone product 3b formed by integration of HMFO and LmrR_V15pAF_RMH in vivo. (a) At wavelength $336 \mathrm{~nm}$. (b) At wavelength $500 \mathrm{~nm}$.

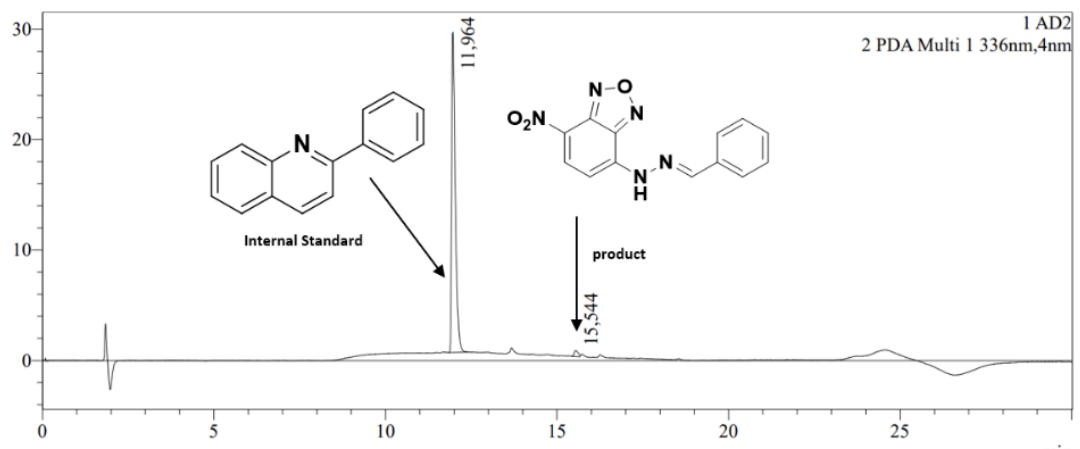

b

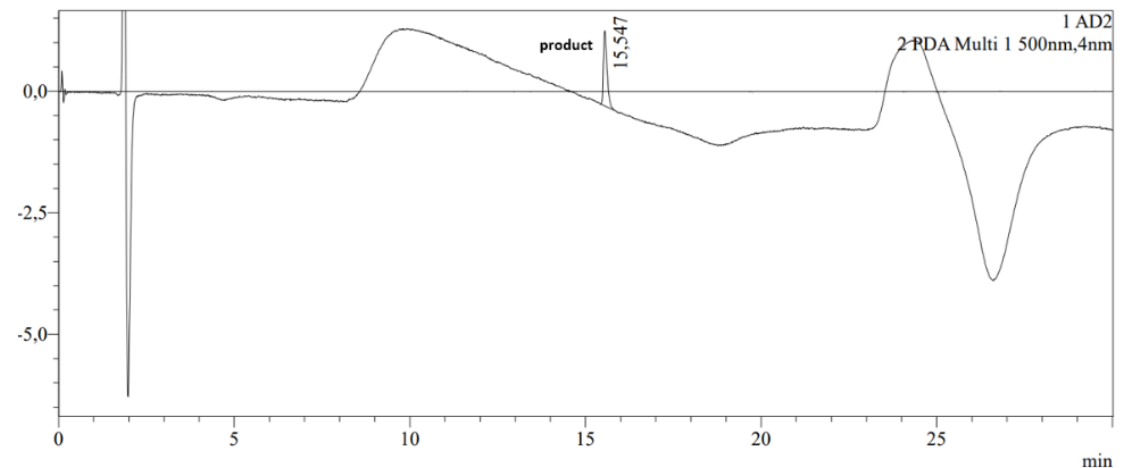

Figure S14. HPLC chromatogram of Hydrazone product $3 \mathbf{b}$ in the presence only HMFO in vivo (i.e. in absence of LmrR_V15pAF_RMH). (a) At wavelength $336 \mathrm{~nm}$. (b) At wavelength is $500 \mathrm{~nm}$. 
a

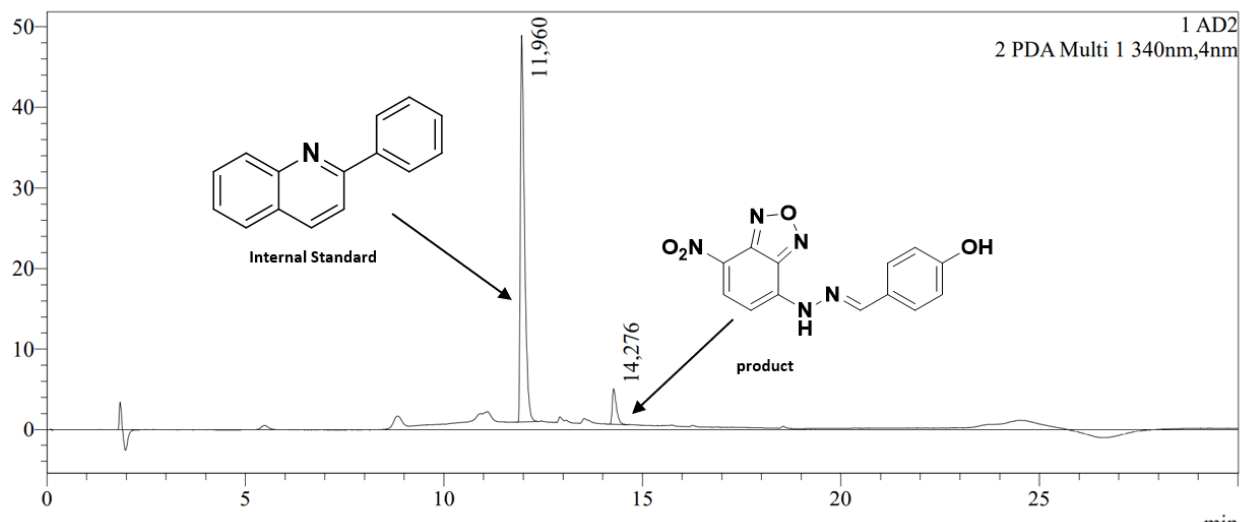

b

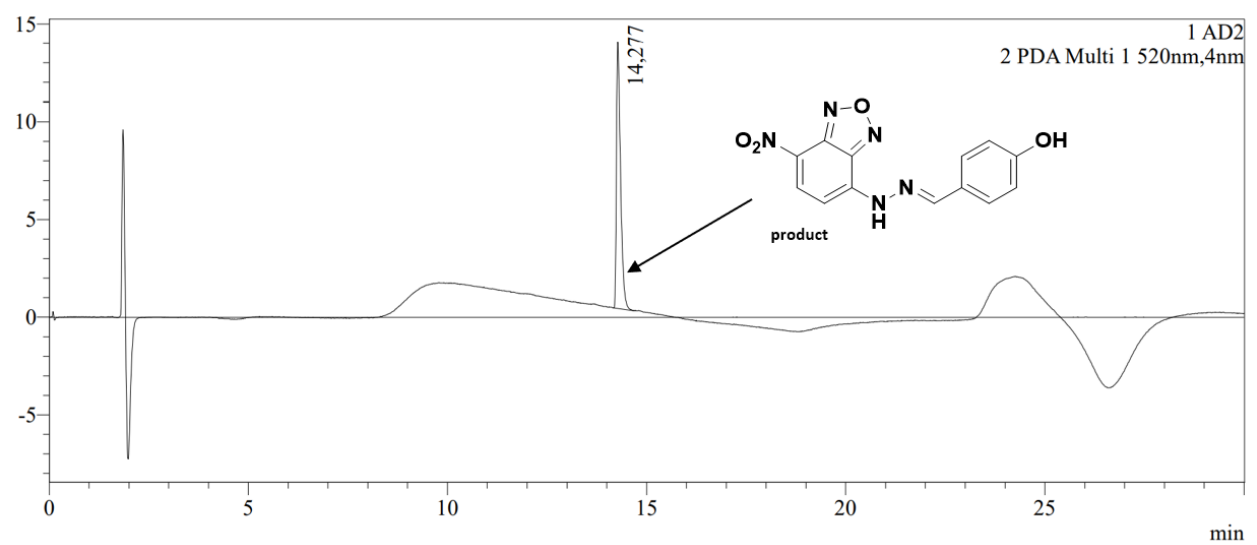

Figure S15. HPLC chromatogram of Hydrazone product 3a formed by integration of HMFO and LmrR_V15pAF_RMH in vivo. (a) At wavelength $340 \mathrm{~nm}$. (b) At wavelength is $520 \mathrm{~nm}$. 


\section{Recycling Experiment}

a

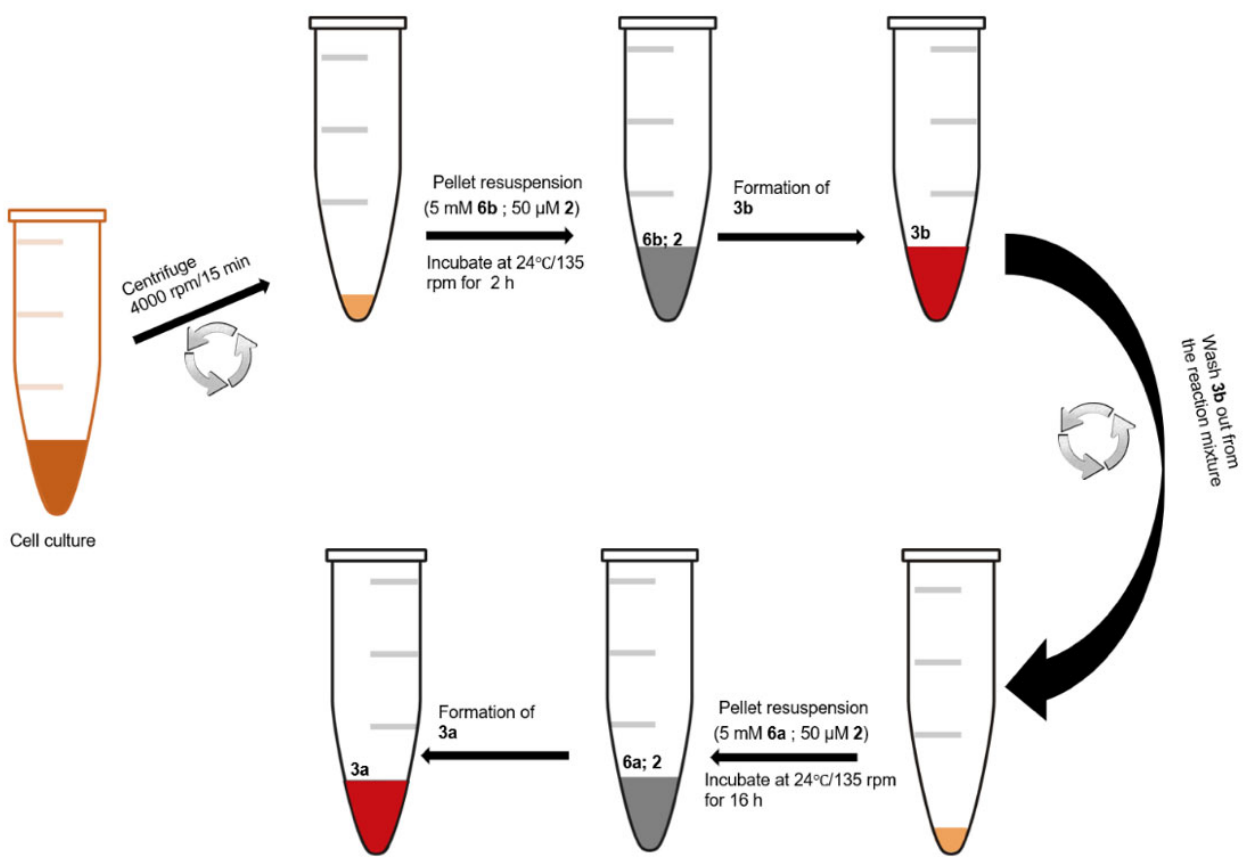

b

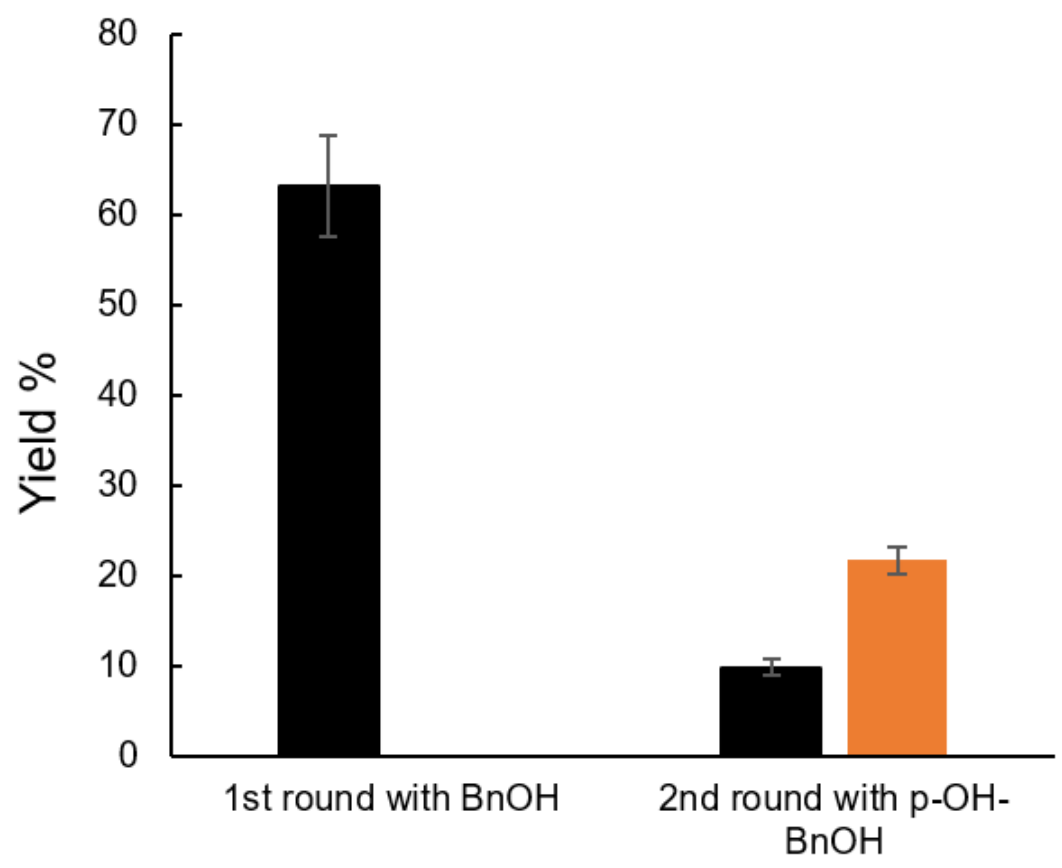

Figure S16. Recycling experiment to establish that catalysis takes place in the cell and the cell remains intact during and after catalysis (a) Schematic workflow of an in vivo recycling experiment of hydrazone formation reaction using HMFO/LmrR_V15pAF_RMH. Products $(\mathbf{3} \mathbf{a}, \mathbf{b})$ were analyzed with the HPLC after extraction. (b) Product yields detected after each catalysis experiment, first round of reaction resulted in $63 \%$ of $3 \mathbf{b}$ (black) and the second round yielded $22 \%$ of $3 a$ (orange) with $10 \%$ residual $\mathbf{3 a}$. 


\section{Cell viability test after catalysis}

After the in vivo catalysis, reaction mixtures were spun down at $8000 \mathrm{rpm}$ for $30 \mathrm{mins}$ and resuspended in LB medium. Serial dilutions $\left(10 \mathrm{x}-10^{-8}\right)$ of selected samples were plated on LB plates containing $100 \mu \mathrm{g} / \mathrm{mL}$ ampicillin, $10 \mu \mathrm{g} / \mathrm{mL}$ spectinomycin and 50 $\mu \mathrm{g} / \mathrm{mL}$ Kanamycin for 3 plasmid system and plates containing $100 \mu \mathrm{g} / \mathrm{mL}$ ampicillin and $10 \mu \mathrm{g} / \mathrm{mL}$ spectinomycin for 2 plasmid system. Plates were then incubated at 37 ${ }^{\circ} \mathrm{C}$ overnight. The following day, colonies were counted, plates with 10x diluted were confluent and therefore not countable. However plates with dilutions from $10^{-5}$ were countable.

a

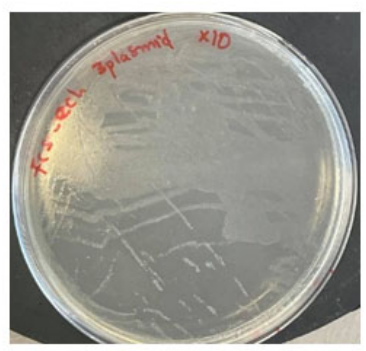

b

FCS_ECH 3-plasmid $\times 10^{-5}$

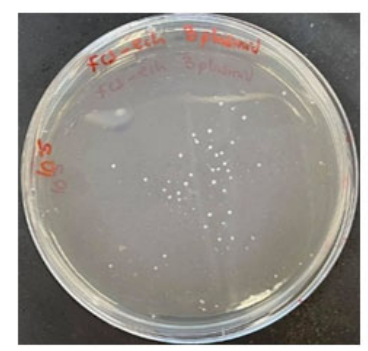

HMFO 2-plasmid

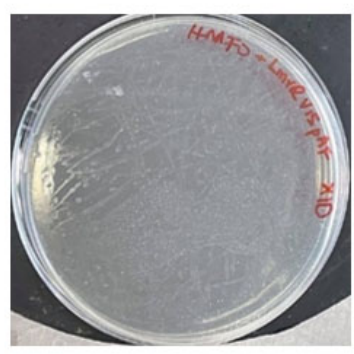

HMFO 2-plasmid $\times 10^{-6}$

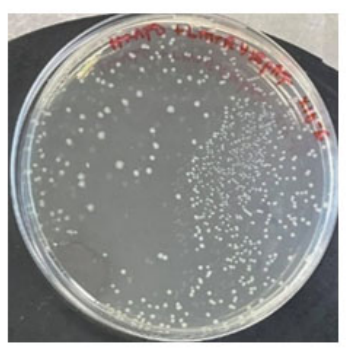

CAR_SFP 3-plasmid

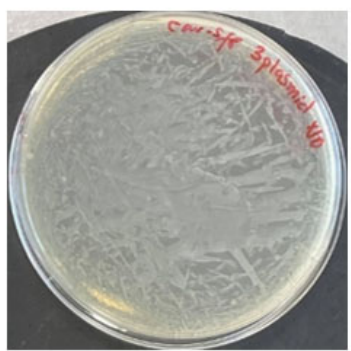

CAR_SFP 3-plasmid $\times 10^{-6}$

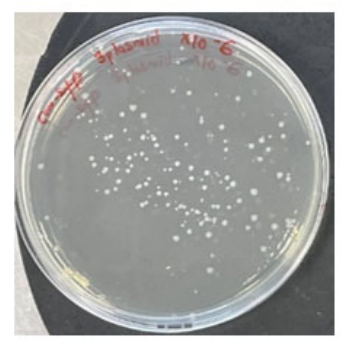

Figure S17. Cell viability test after hydrazone formation reaction. (a) Image of the confluency of colonies after 10 times dilution. (b) Plate with FCS_ECH 3-plasmid had 45 colonies, HMFO 2-plasmid had 336 colonies and CAR_SFP 3-plasmid had 141 colonies after several serial dilutions.

After the viability test, LmrR_V15pAF_RMH/HMFO system was used to repeat the cell viability test and this time with 2 different substrates. After protein expression at $24^{\circ} \mathrm{C} / 135$ 
rpm for 48 hours, cultures were collected and spun down at $4000 \mathrm{rpm}$ for 10 mins. The pellet was resuspended in $50 \mathrm{mM}$ potassium phosphate buffer at $\mathrm{pH} 6.5$, reaction was performed by adding $5 \mathrm{mM}$ of benzylalcohol and $50 \mu \mathrm{M}$ of NBD-H to the cell and incubated at $24^{\circ} \mathrm{C} / 135$ rpm for 2 hours. The reaction mixtures were then spun down and the cells resuspended in LB media, serial dilutions were done down to $10^{-6}$ and $200 \mu$ was plated on LB agar each dilution and incubated at $37^{\circ} \mathrm{C}$ overnight. A single colony from the $10^{-6}$ dilution plate was picked and grown in LB media while expressing LmrR_V15pAF_RMH/HMFO. Catalysis was performed as previously described with the new cells, now using $5 \mathrm{mM}$ p-hydroxybenzyl alcohol and 50 $\mu \mathrm{M} N B D H$ as substrates in $50 \mathrm{mM}$ potassium phosphate buffer at $\mathrm{pH}$ 6.5. After overnight reaction at $24^{\circ} \mathrm{C} / 135 \mathrm{rpm}$, the samples were again spun down, resuspended in LB and serial dilutions down to $10^{-9}$ were plated on agar. Colonies on the plates with $10^{-8}$ and $10^{-9}$ dilutions yielded 419 and 60 colonies respectively.

a

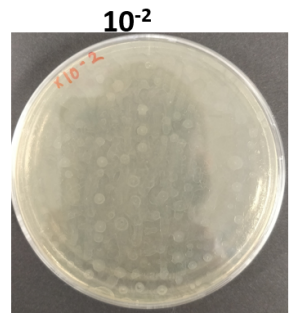

b

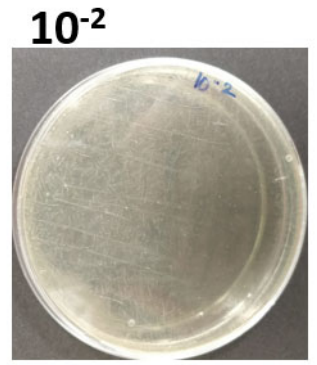

$10^{-6}$

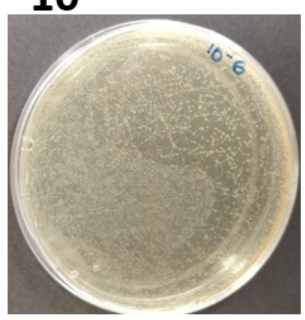

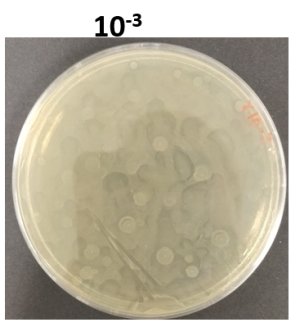

$10^{-3}$

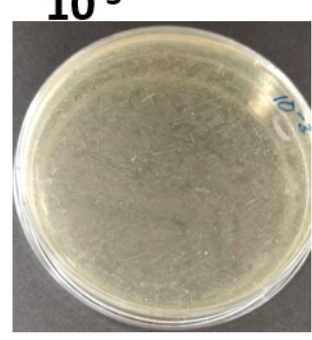

$10^{-7}$

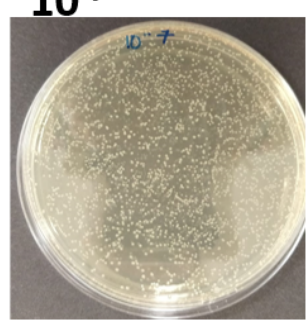

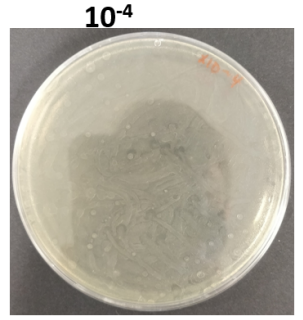
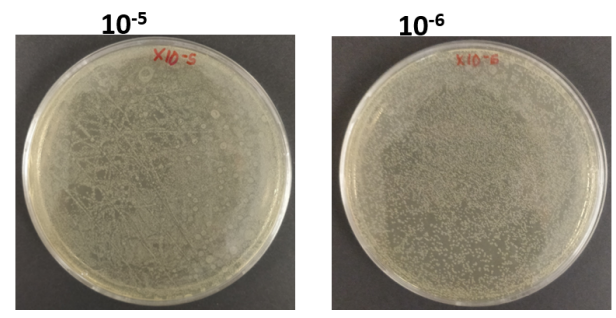

$10^{-4}$

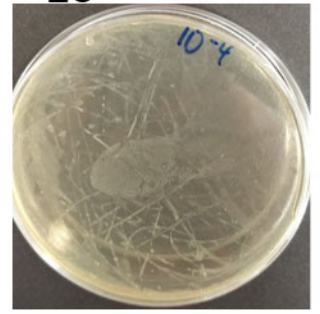

$10^{-5}$

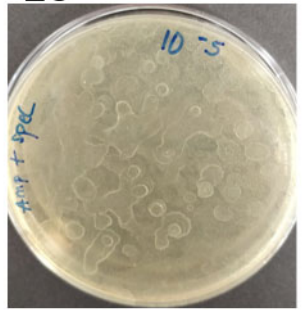

$10^{-8}$

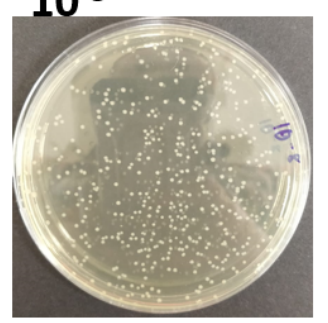

$10^{-9}$

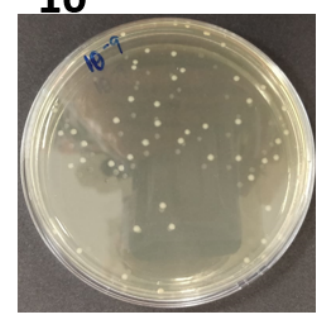

Figure S18. Agar plates with colonies after viability test with LmrR_V15pAF_RMH/HMFO system (a) images of colony plates after the first round of catalysis with $5 \mathrm{mM}$ of benzylalcohol and $50 \mu \mathrm{M}$ of NBD-H for 2 hours. (b)Colony plates after second round of catalysis $5 \mathrm{mM}$ p-hydroxybenzyl alcohol and $50 \mathrm{uM} \mathrm{NBDH}$ for overnight. Colonies counted on the $10^{-8}$ and $10^{-9}$ plates were estimated to be $6 \times 10^{7}$ cells $/ \mathrm{ml}$ for plate $10^{-8}$ and $2 \times 10^{7}$ cells $/ \mathrm{ml}$ for plate $10^{-9}$ viable cells after catalysis, compared to $1.92 \times 10^{10}$ cells $/ \mathrm{ml}$ at the onset. 


\section{Gene sequence used in the work}

\section{LmrR-V15TAG RMH}

ATGGGTGCCGAAATCCCGAAAGAAATGCTGCGTGCTCAAACCAATTAGATCCTGCTGATGGTCCTGAAACAA GGCGATAACTATGTGTATGGCATTATCAAACAGGTGAAAGAAGCGAGCAACGGTGAAATGGAACTGAATGAA GCCACCCTGTATACGATTTTTGATCGTCTGGAACAGGACGGCATTATCAGCTCTTACTGGGGTGATGAAAGT CAAGGCGGTCGTCGCAAATATTACCGTCTGACCGAAATCGGCCATGAAAACATGCGCCTGCGGCATGAATC CTGGAGTCGTGTGGACAAAATCATTGAAAATCTGGAAGCAAACAAAAAATCTGAAGCGATCAAATCTAGAGG TGGCAGCGGTGGCTGGAGCCACCCGCAGTTCGAAAAATAA

\section{HMFO}

ATGACTGATACGATTTTTGACTACGTGATTGTTGGCGGTGGCACGGCGGGTAGCGTTCTGGCCAACCGTCT GTCCGCCCGTCCGGAGAATCGCGTGTTGCTGATTGAGGCCGGTATTGATACCCCGGAAAACAATATTCCGC CGGAGATCCACGATGGCCTGCGCCCGTGGCTGCCGCGTCTGAGCGGTGATAAGTTCTTTTTGGCCGAATCT GACCGTCTACCGTGCCGCGGAACACCCGGGTATCACGCGCGAGCCGCAGTTCTATGAACAAGGCCGTCTG CTGGGCGGTGGTAGCAGCGTGAACATGGTCGTTTCTAACCGTGGTCTGCCTCGCGACTATGACGAATGGCA GGCACTGGGCGCAGATGGTTGGGATTGGCAGGGTGTTCTGCCGTACTTCATCAAGACCGAGCGTGACGCG GACTACGGTGACGACCCGTTGCATGGCAATGCGGGTCCGATTCCGATCGGTCGCGTCGATTCGCGTCACT GGAGCGACTTCACGGTGGCGGCAACCCAAGCTCTGGAAGCGGCTGGCCTGCCGAACATTCACGACCAAAA CGCACGTTTTGATGACGGTTACTTCCCACCGGCATTTACGTTGAAAGGTGAAGAGCGCTTCAGCGCCGCAC GCGGTTATCTGGATGCGAGCGTCCGTGTGCGTCCGAACCTGAGCCTGTGGACTGAGAGCCGTGTCCTGAA GCTGCTGACCACTGGCAATGCAATCACCGGTGTGAGCGTGCTGCGTGGTCGCGAAACCCTGCAAGTTCAAG CGCGCGAGGTCATCCTGACCGCCGGTGCGTTGCAAAGCCCAGCGATTCTGTTGCGCACCGGCATCGGCCC TGCGGCGGATCTGCACGCACTGGGTATTCCTGTTCTGGCAGACCGTCCGGGTGTTGGTCGCAATCTGTGG GAGCACAGCTCTATCGGTGTGGTTGCCCCGCTGACCGAGCAGGCACGTGCAGACGCCAGCACGGGTAAAG CCGGCTCTCGCCATCAACTGGGTATCCGTGCGTCGTCCGGCGTAGATCCGGCGACGCCTAGCGACCTGTT TCTGCATATCCATGCTGATCCAGTCAGCGGTCTGGCAAGCGCTCTGTTCTGGGTGAACAAGCCAAGCTCCA CCGGCTGGCTGAAGCTGAAGGACGCGGACCCGTTTAGCTACCCGGACGTAGACTTCAATCTGCTGAGCGAT CCGCGCGACTTGGGTCGTCTGAAAGCGGGCCTGCGTCTGATCAAACATTACTTCGCATATCCGTCCCTGGC GAAATATGGTTTGGCGCTGGCATTGAGCCGTTTTGAGGCACCGCAGCCGGGTGGTCCGCTGCTGAACGAC CTGTTGCAGGACGAAGCCGCCCTGGAACGCTATTTGCGTACGAACGTCGGCGGTGTTTGGCATGCGAGCG GCACGGCGCGTATCGGCCGTGCGGATGATTCCCAGGCTGTTGTCGATAAAGCGGGTCGTGTGTACGGCGT CACCGGCCTGCGTGTTGCGGACGCAAGCATTATGCCGACCGTTCCGACCGCCAATACCAATCTGCCGACG CTGATGCTGGCTGAGAAAATTGCGGATGCGATTCTGACCCAGGCTTAA

\section{CAR-SFP}

CCATGGGCAGCAGCCATCACCATCATCACCACAGCCAGGATCCGAATTCGATGGCTGTGGACTCGCCGGAT GAACGCCTGCAACGCCGTATCGCCCAACTGTTTGCCGAAGATGAACAAGTGAAAGCTGCCCGCCCGCTGGA AGCAGTTAGCGCGGCCGTCTCTGCACCGGGTATGCGTCTGGCTCAGATCGCAGCTACGGTGATGGCTGGT TATGCGGATCGTCCGGCGGCGGGCCAGCGTGCTTTCGAACTGAATACCGATGACGCAACCGGCCGTACCA GCCTGCGTCTGCTGCCGCGTTTTGAAACCATTACGTACCGCGAACTGTGGCAGCGTGTCGGCGAAGTGGCA GCTGCGTGGCATCACGACCCGGAAAACCCGCTGCGTGCGGGTGATTTTGTGGCCCTGCTGGGCTTCACCA GCATTGATTATGCAACGCTGGATCTGGCTGACATCCATCTGGGTGCGGTTACCGTGCCGCTGCAAGCGAGC GCGGCGGTGTCCCAACTGATTGCAATCCTGACCGAAACGAGTCCGCGCCTGCTGGCGTCCACCCCGGAAC ATCTGGATGCTGCGGTGGAATGCCTGCTGGCAGGCACCACGCCGGAACGTCTGGTGGTTTTCGATTATCAC CCGGAAGATGACGATCAGCGCGCCGCATTTGAAAGTGCGCGTCGCCGTCTGGCAGATGCAGGTTCCCTGG TGATCGTTGAAACCCTGGACGCGGTGCGTGCGCGTGGCCGTGATCTGCCGGCTGCGCCGCTGTTTGTCCC GGATACCGACGATGACCCGCTGGCGCTGCTGATTTATACGTCAGGTTCGACCGGCACGCCGAAAGGTGCC ATGTACACCAATCGTCTGGCCGCAACGATGTGGCAGGGCAACTCAATGCTGCAAGGCAACAGCCAACGCGT TGGCATTAACCTGAATTATATGCCGATGAGTCATATTGCGGGTCGTATCTCCCTGTTCGGCGTGCTGGCGCG TGGCGGCACCGCATACTTTGCTGCGAAATCAGACATGAGCACCCTGTTTGAAGATATTGGCCTGGTTCGCC 
CGACCGAAATCTTTTTCGTTCCGCGTGTCTGTGACATGGTGTTTCAGCGCTATCAAAGCGAACTGGATCGCC GTTCTGTCGCTGGTGCGGATCTGGACACCCTGGACCGCGAAGTGAAAGCGGATCTGCGTCAGAATTACCTG GGCGGTCGCTTCCTGGTTGCAGTCGTGGGCTCGGCTCCGCTGGCCGCAGAAATGAAAACGTTTATGGAAA GCGTGCTGGACCTGCCGCTGCATGATGGTTATGGCAGTACCGAAGCCGGCGCATCCGTTCTGCTGGATAAC CAGATCCAACGTCCGCCGGTCCTGGACTATAAACTGGTCGATGTGCCGGAACTGGGTTACTTTCGCACGGA TCGTCCGCACCCGCGTGGCGAACTGCTGCTGAAAGCAGAAACCACGATTCCGGGTTATTACAAACGCCCGG AAGTTACGGCGGAAATCTTTGATGAAGACGGCTTCTATAAAACCGGCGATATTGTGGCCGAACTGGAACATG ACCGCCTGGTTTACGTGGATCGTCGTAACAATGTTCTGAAACTGTCCCAGGGCGAATTTGTGACCGTTGCGC ACCTGGAAGCTGTGTTCGCGAGCAGCCCGCTGATCCGTCAAATTTTTATCTATGGTAGTTCCGAACGCAGTT ACCTGCTGGCCGTCATTGTGCCGACCGATGACGCACTGCGTGGCCGCGATACCGCTACGCTGAAAAGCGC TCTGGCGGAATCTATTCAGCGTATCGCCAAAGACGCAAATCTGCAACCGTATGAAATTCCGCGCGATTTTCT GATCGAAACCGAACCGTTCACGATTGCCAATGGCCTGCTGAGCGGTATCGCAAAACTGCTGCGCCCGAACC TGAAAGAACGTTATGGTGCGCAGCTGGAACAAATGTACACCGACCTGGCTACGGGCCAGGCAGATGAACTG CTGGCCCTGCGCCGTGAAGCTGCGGATCTGCCGGTGCTGGAAACCGTTAGCCGTGCCGCAAAAGCGATGC TGGGTGTGGCAAGCGCGGATATGCGTCCGGACGCACATTTTACCGATCTGGGCGGTGACAGCCTGTCTGC ACTGAGTTTTTCCAACCTGCTGCACGAAATCTTCGGTGTTGAAGTCCCGGTGGGTGTTGTCGTGTCTCCGGC AAACGAACTGCGTGATCTGGCGAATTATATTGAAGCCGAACGCAACAGTGGCGCAAAACGTCCGACCTTCA CGTCAGTGCATGGCGGTGGCTCGGAAATTCGTGCTGCGGATCTGACCCTGGACAAATTTATCGATGCACGC ACGCTGGCCGCAGCTGATTCTATTCCGCACGCCCCGGTGCCGGCACAGACCGTTCTGCTGACGGGTGCGA ATGGCTATCTGGGTCGTTTCCTGTGCCTGGAATGGCTGGAACGCCTGGATAAAACCGGCGGCACCCTGATT TGTGTTGTCCGTGGTAGCGACGCGGCGGCGGCACGTAAACGTCTGGATTCAGCCTTTGATAGCGGCGATC CGGGCCTGCTGGAACATTATCAGCAACTGGCAGCACGTACCCTGGAAGTGCTGGCAGGCGATATTGGTGAC CCGAACCTGGGCCTGGATGACGCGACCTGGCAGCGTCTGGCAGAAACGGTCGATCTGATTGTGCATCCGG CAGCTCTGGTGAATCACGTTCTGCCGTACACCCAGCTGTTTGGCCCGAACGTGGTTGGCACCGCGGAAATT GTGCGCCTGGCTATCACCGCGCGTCGTAAACCAGTGACCTATCTGTCTACGGTTGGCGTCGCAGATCAGGT TGACCCGGCTGAATACCAAGAAGATAGCGATGTGCGTGAAATGTCTGCGGTGCGTGTCGTGCGCGAAAGCT ATGCCAACGGTTACGGCAATTCTAAATGGGCTGGTGAAGTGCTGCTGCGCGAAGCGCATGATCTGTGCGGT CTGCCGGTGGCAGTTTTTCGTTCAGATATGATTCTGGCACACTCGCGCTATGCTGGTCAGCTGAATGTCCAA GATGTGTTCACCCGTCTGATTCTGTCACTGGTTGCTACGGGCATCGCGCCGTATTCGTTTTACCGCACCGAT GCAGACGGTAACCGTCAGCGCGCCCATTACGATGGTCTGCCGGCAGATTTCACCGCGGCGGCGATTACGG CGCTGGGTATCCAGGCCACCGAAGGCTTTCGCACGTATGATGTGCTGAATCCGTATGATGACGGTATTAGT CTGGACGAATTTGTTGATTGGCTGGTCGAATCCGGCCATCCGATTCAGCGTATCACGGATTATTCAGACTGG TTTCACCGCTTCGAAACCGCCATCCGTGCACTGCCGGAAAAACAGCGTCAAGCCAGCGTGCTGCCGCTGCT GGATGCATACCGTAACCCGTGTCCGGCCGTTCGCGGTGCAATTCTGCCGGCTAAAGAATTTCAGGCTGCGG TCCAAACCGCGAAAATTGGCCCGGAACAGGATATTCCGCACCTGAGTGCCCCGCTGATTGATAAATACGTG TCTGACCTGGAACTGCTGCAACTGCTGGGTAGTGGCTCTGGACTGGTGGGTGCCCTGATGCACGTGATGCA GAAGCGCAGCCGCGCCATCCACTCCTCCGACGAAGGGGAGGACCAGGCTGGCGATGAAGATGAAGATTGA $\underline{\text { GCGGCCGC }}$

\section{FSC}

GGATCCGGTGCGTAACCAGGGTCTGGGTTCTTGGCCGGTTCGTCGTGCTCGTATGTCTCCGCACGCTACCG CTGTTCGTCACGGTGGTACCGCTCTGACCTACGCTGAACTGTCTCGTCGTGTTGCTCGTCTGGCTCACGGT CTGCGTGAAGCTGGTGTTCGTCCGGGTGACCGTGTTGCTTACCTGGGTCCGAACCACCCGGCTTACCTGGA AACCCTGTTCGCTTGCGGTCAGGCTGGTGCTGTTTTCGTTCCGCTGAACTTCCGTCTGGGTGTTCCGGAACT GGACCACGCTCTGGCTGACTCTGGTGCTTCTGTTCTGATCCACACCCCGGAACACGCTGAAACCGTTGCTG 
CTCTGGCTGGTGACCGTCTGCTGCGTGTTCCGGCTGGTGAACTGGAAGCTGCTGACGACGAACCGCTGGA CCTGCCGGTTGGTCTGGACGACGTTTGCCTGCTGATGTACACCTCTGGTTCTACCGGTCGTCCGAAAGGTG CTATGCTGACCCACGGTAACCTGACCTGGAACTGCGTTAACGTTCTGGTTGAAACCGACCTGGCTTCTGAC GAACGTGCTCTGGTTGCTGCTCCGCTGTTCCACGCTGCTGCTCTGGGTATGGTTTGCCTGCCGACCCTGCT GAAAGGTGGTACCGTTATCCTGCACTCTGCTTTCGACCCGGGTGCTGTTCTGTCTGCTGTTGAACAGGAAC GTGTTACCCTGGTTTTCGGTGTTCCGACCATGTACCAGGCTATCGCTGCTCACCCGCGTTGGCGTTCTACC GACCTGTCTTCTCTGCGTACCCTGCTGTGCGGTGGTGCTCCGGTTCCGGCTGACCTGGCTGGTCGTTACCT GGACCGTGGCCTGGCGTTCGTTCAGGGTTACGGTATGACCGAAGCTGCTCCGGGTGTTCTGGTTCTGGAC CGTGCTCACGTTGCTGAAAAAATCGGTTCTGCTGGTGTTCCGTCTTTCTTCACCGACGTTCGTGTTGCTGGT CCGTCTGGTGAACCGGTTCCGCCGGGTGAAAAAGGTGAAATCGTTGTTTCTGGTCCGAACGTTATGAAAGG TTACTGGGGTCGTCCGGAAGCTACCGCTGAAGTTCTGCGTGACGGTTGGTTCCGTTCTGGTGACGTTGCTA CCGTTGACGGTGACGGTTACTTCCACGTTGTTGACCGTCTGAAAGACATGATCATCTCTGGTGGTGAAAACA TCTACCCGGCTGAAGTTGAAAACGAACTGTACGGTTACCCGGGTGTTGAAGCTTGCGCTGTTATCGGTGTTC CGGACCCGCGTTGGGGTGAAGTTGGTAAAGCTGTTGTTGTTCCGGCTGCTGGTTCTCGTATCGACGGTGCT GAACTGCTGGCTTGGCTGCGTACCCGTCTGGCTGGTTACAAAGTTCCGAAATCTGTTGAATTCACCGACCGT CTGCCGACCACCGGTTCTGGTAAAATCCTGAAAGGTGAAGTTCGTCGTCGTTTCGGTTAAGCGGCCGC

\section{$E C H$}

CATATGTCTACCGCTGTTGGTAACGGTCGTGTTCGTACCGAACCGTGGGGTGAAACCGTTCTGGTTGAATTC GACGAAGGTATCGCTTGGGTTACCCTGAACCGTCCGGACAAACGTAACGCTATGAACCCGACCCTGAACGA CGAAATGGTTCGTGTTCTGGACCACCTGGAAGGTGACGACCGTTGCCGTGTTCTGGTTCTGACCGGTGCTG GTGAATCTTTCTCTGCTGGTATGGACCTGAAAGAATACTTCCGTGAAGTTGACGCTACCGGTTCTACCGCTG TTCAGATCAAAGTTCGTCGTGCTTCTGCTGAATGGCAGTGGAAACGTCTGGCTAACTGGTCTAAACCGACCA TCGCTATGGTTAACGGTTGGTGCTTCGGTGGTGCTTTCACCCCGCTGGTTGCTTGTGACCTGGCGTTCGCT GACGAAGACGCTCAGTTCGGTCTGTCTGAAGTTAACTGGGGTATCCCACCGGGTGGCGTTGTAAGCCGTGC TCTGGCTGCTACCGTTCCGCAGCGTGACGCTCTGTACTACATCATGACCGGTGAACCGTTCGACGGTCGTC GTGCTGCTGAAATGCGTCTGGTTAACGAAGCTCTGCCGGCTGACCGTCTGCGTGAACGTACCCGTGAAGTT GCTCTGAAACTGGCTTCTATGAACCAGGTTGTTCTGCACGCTGCTAAAACCGGTTACAAAATCGCTCAGGAA ATGCCGTGGGAACAGGCTGAAGACTACCTGTACGCTAAACTGGACCAGTCTCAGTTCGCTGACAAAGCTGG TGCTCGTGCTAAAGGTCTGACCCAGTTCCTGGACCAGAAATCTTACCGTCCGGGTCTGTCTGCTTTCGACCC GGAAAAATAACTCGAG

\section{Note}

All underlined sequence are the restriction sites used for cloning 


\section{Calibration Curves for Aldehydes}

Benzaldehyde (1b) IS: 2-methylanisole

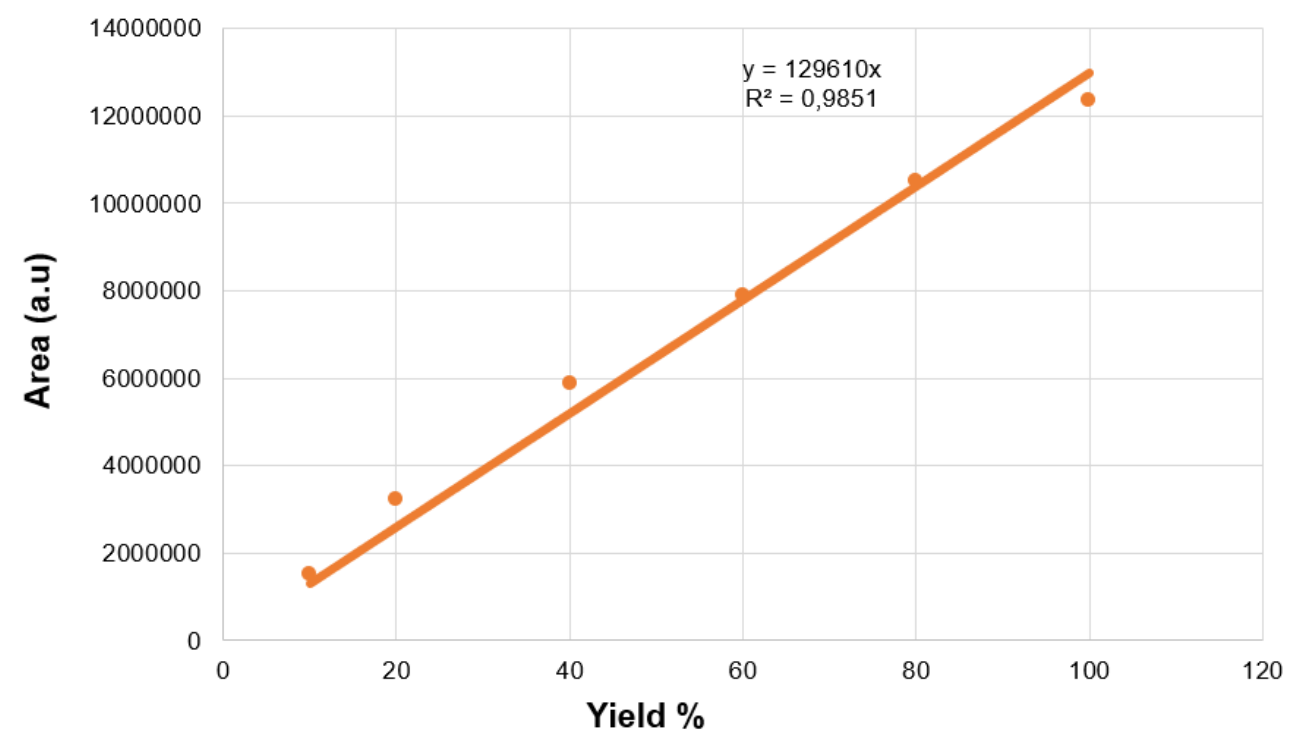

p-OH Benzaldehyde (1a) IS: (2-Phenylquinoline)

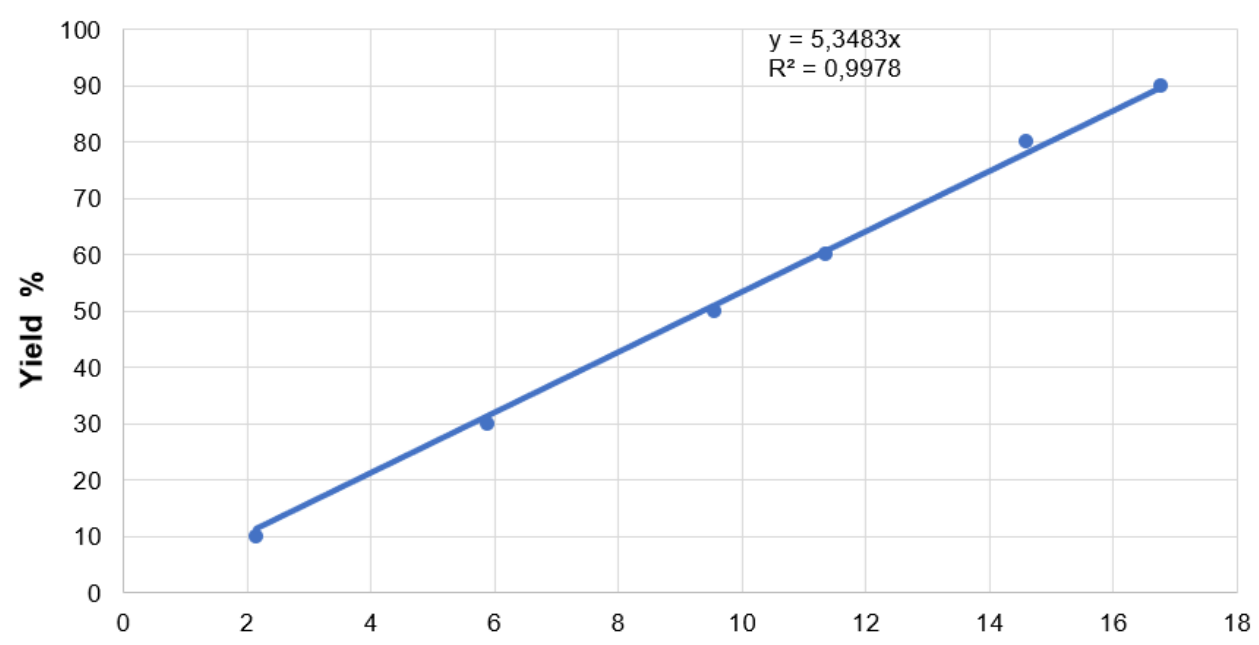

Product ratio / IS 
Chemical synthesis and calibration curves of hydrazone products $3 \mathrm{a}$ and $\mathbf{3 b}$.

The hydrazone product between NBD-H and 4-hydroxybenzaldehyde (4-HBA) was prepared according to the same procedure as we previously reported. ${ }^{[3]}$ The hydrazone product of the reaction between NBD-H and benzaldehyde was prepared as previously described in the literature. ${ }^{[4]}$ In brief, NBDH ( $25 \mathrm{mg}, 0.13 \mathrm{mmol}, 1$ equiv) was dissolved in methanol $(10 \mathrm{~mL})$. Benzaldehyde $(0.13 \mathrm{~mL}, 1.3 \mathrm{mmol}, 10$ equiv) was added, turning the reaction mixture a red color. The product gradually formed as a dark red-black precipitate and was isolated by vacuum filtration. ${ }^{1} \mathrm{H} \mathrm{NMR}(400 \mathrm{MHz}$, $\left.\left(\mathrm{CD}_{3}\right)_{2} \mathrm{CO}\right): 8.62(\mathrm{~d}, 1 \mathrm{H}, 3 \mathrm{~J}=8.8 \mathrm{~Hz}), 8.59(\mathrm{~s}, 1 \mathrm{H}), 7.92-7.79(\mathrm{~m}, 2 \mathrm{H}), 7.56-7.39(\mathrm{~m}$, $3 \mathrm{H}), 7.31(\mathrm{~d}, 1 \mathrm{H}, 3 \mathrm{~J}=8.8 \mathrm{~Hz})$
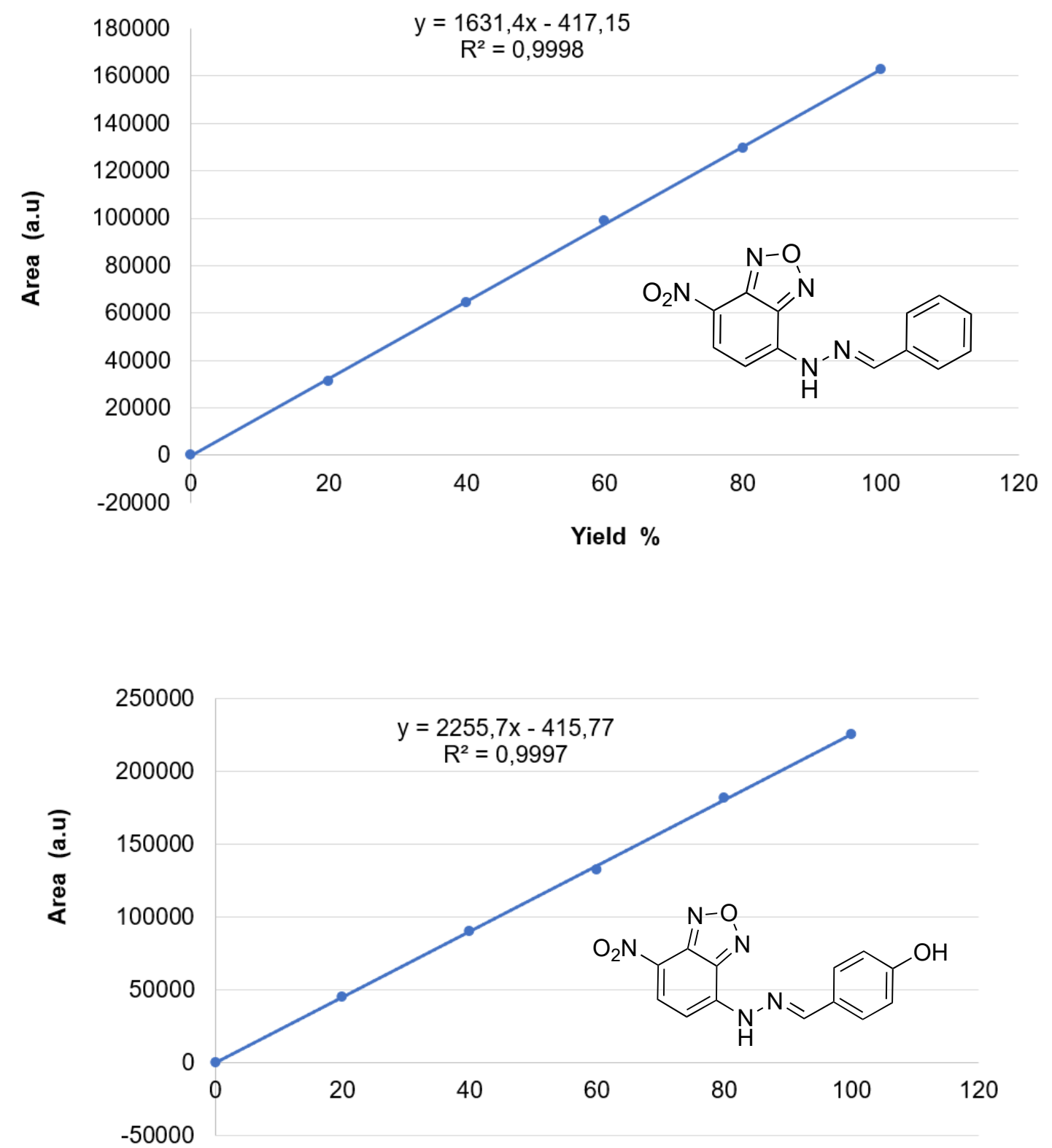

Yield \% 


\section{NMR spectra}

$1 \mathrm{H}-\mathrm{NMR}$ product $\mathbf{3 b}$ in acetone-d6

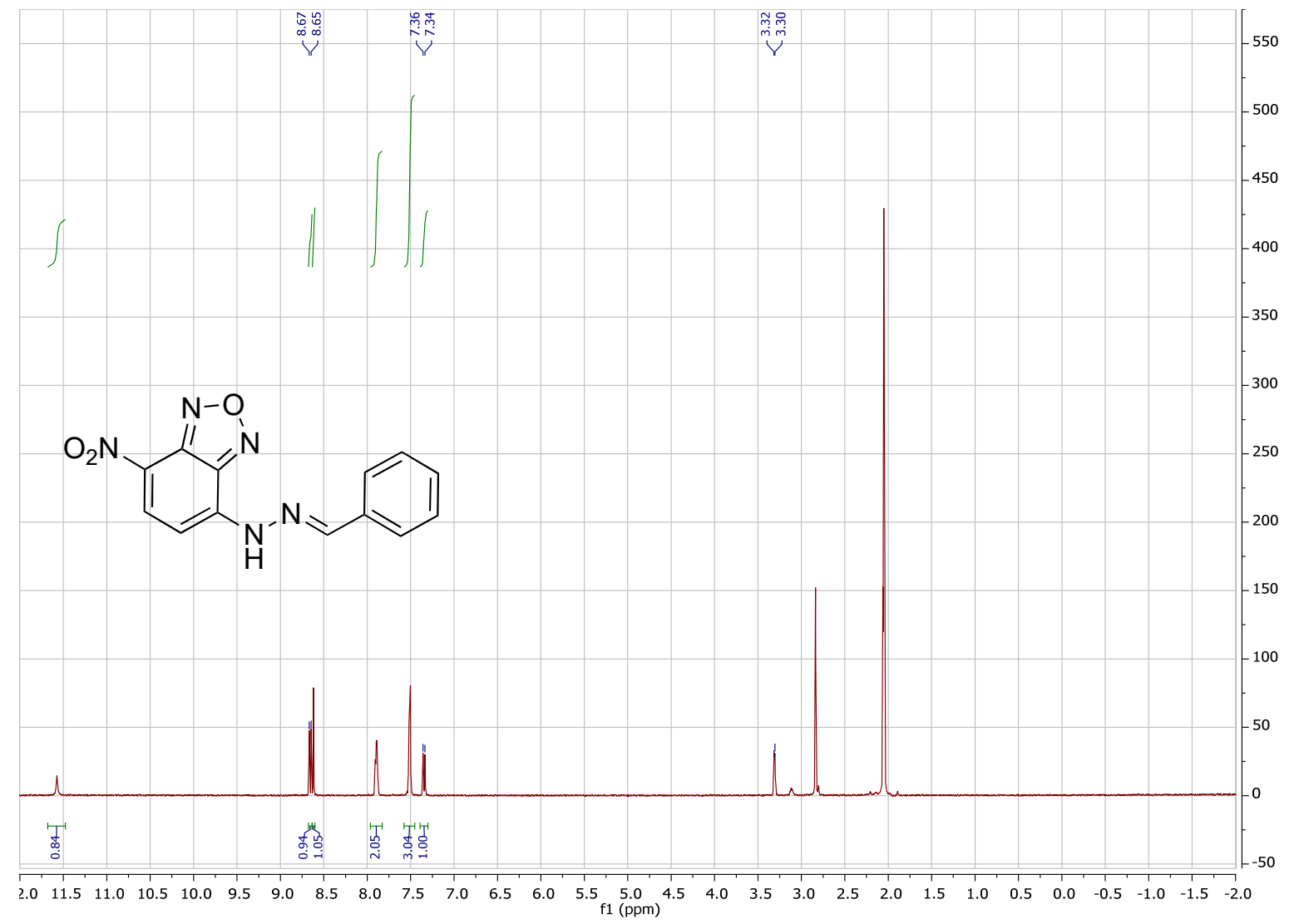

\section{References}

[1] R. A. Mehl, J. C. Anderson, S. W. Santoro, L. Wang, A. B. Martin, D. S. King,

D. M. Horn, P. G. Schultz, J. Am. Chem. Soc. 2003, 125, 935-939.

[2] C. Mayer, C. Dulson, E. Reddem, A. M. W. H. Thunnissen, G. Roelfes, Angew. Chemie - Int. Ed. 2019, 58, 2083-2087.

[3] I. Drienovská, C. Mayer, C. Dulson, G. Roelfes, Nat. Chem. 2018, 10, 946952.

[4] J. A. Key, C. Li, C. W. Cairo, Bioconjugate Chem. 2012, 23, 363-371. 